\title{
Absorption Spectra of Water Vapor and Carbon Dioxide in the Region of 2.7 Microns
}

\author{
W. S. Benedict and Earle K. Plyler
}

\begin{abstract}
The absorption due to atmospheric $\mathrm{H}_{2} \mathrm{O}$ and $\mathrm{CO}_{2}$ in the region of $2.7 \mu$ has been remeasured under higher resolution than previously reported. By using a 15,000-line grating and a lead sulfide photoconductive cell, some 450 lines have been measured between 2.43 microns $\left(4,090 \mathrm{~cm}^{-1}\right)$ and $2.87 \mu\left(3,500 \mathrm{~cm}^{-1}\right)$. A rotational analysis may be given for nearly all the lines. In addition to the known $\nu_{3}$ fundamental of $\mathrm{H}_{2} \mathrm{O}$ which causes the strongest absorption in the region, a number of lines due to the $\nu_{1}$ fundamental of $\mathrm{H}_{2} \mathrm{O}$, and the $\left\{\begin{array}{ll}02^{\circ} & 1 \\ 10^{\circ} & 1\end{array}\right\}$ combination bands of $\mathrm{CO}_{2}$ may be recognized. The $\nu_{1}$ analysis does not agree with one suggested previously. The following are the principal molecular constants derived from the new analyses:
\end{abstract}

\begin{tabular}{|c|c|c|c|c|c|c|c|}
\hline Molecule & & $v_{2}$ & $v_{3}$ & $\nu_{0}$ & $A$ & $B$ & $C$ \\
\hline $\begin{array}{l}\mathrm{H}_{2} \mathrm{O}_{\ldots} \ldots \ldots \\
\mathrm{H}_{2} \mathrm{O}_{\ldots} \ldots \ldots \\
\mathrm{CO}_{2 \ldots} \ldots \ldots \\
\mathrm{CO}_{2 \ldots} \ldots \ldots \ldots\end{array}$ & $\begin{array}{l}0 \\
1 \\
0 \\
1\end{array}$ & $\begin{array}{l}0 \\
0 \\
2 \\
0\end{array}$ & $\begin{array}{l}1 \\
0 \\
1 \\
1\end{array}$ & $\begin{array}{c}\mathrm{cm}^{-1} \\
3755.79 \\
3657.05 \\
3612.91 \\
3714.59\end{array}$ & $\begin{array}{c}\mathrm{cm}^{-1} \\
26.64 \\
27.13 \\
\end{array}$ & \begin{aligned} \multicolumn{1}{c}{$\mathrm{m}^{-1}$} \\
14.40 \\
14. 29 \\
0. $3875_{8} \\
.3872_{8}\end{aligned}$ & $\begin{array}{l}\mathrm{cm}^{-1} \\
9.16 \\
9.11 \\
\end{array}$ \\
\hline
\end{tabular}

\section{Introduction}

Since the beginning of infrared spectroscopy, the existence of a region of strong atmospheric absorption near $2.7 \mu$ has been recognized. With each advance in experimental techniques, permitting higher resolving power, more and more fine structure has been observed in the absorption band. The lines have the highly irregular spacing characteristic of an asymmetric rotator, and it has long been recognized that the strongest absorption is due to the $\mathrm{H}_{2} \mathrm{O}$ molecule, in a fundamental vibration-rotation band. The first rotational analysis of this molecule was achieved by Mecke [1], ${ }^{1}$ who was able to assign the strongest lines, as resolved by Plyler and Sleator $[2]$, to transitions of low $J$ in the asymmetrical valence vibration, $\nu_{3}$. Nielsen, [3], with improved resolution, made a more extensive analysis of the $\nu_{3}$ band, and also attributed some of the weaker lines at longer wavelength to the symmetrical valence vibration, $\nu_{1}$, which appears strongly in the Raman effect.

In the course of a study [4] of the structure of a large number of the overtone and combination bands of $\mathrm{H}_{2} \mathrm{O}$, it appeared that the reported identification of $\nu_{1}$ in the infrared was questionable. We have accordingly reinvestigated the atmospheric absorption in the $2.7-\mu$ region, making use of a recently developed grating spectrograph of high resolution [5]. In the course of the present investigation, we have arrived at a new identification of $\nu_{1}$, have extended the analysis of $\nu_{3}$ to higher values of $J$, and in addition have identified a number of lines of two combination bands of $\mathrm{CO}_{2}$ [6], which had not previously been studied under high resolution. The present analysis of the spectrum accounts for nearly all of the more than 450 lines that have been observed between $2.43 \mu\left(4,090 \mathrm{~cm}^{-1}\right)$ and $2.87 \mu\left(3,500 \mathrm{~cm}^{-1}\right)$. The resulting energy levels are in good agreement with theoretical considerations and with the observations on the overtone and combination bands.

\section{Experimental Procedure}

The spectrometer, which uses a 15,000 -line, $7 \frac{1}{4}$-in ruled surface as the dispersing element and a leadsulfide photoconductive cell as the receiver, has been described [5]. In order to reach the longest wavelengths desired in this study, it was necessary to use the grating at its extreme angle, thus reducing the intensity of radiation. The sensitivity of the $\mathrm{PbS}$ detector also falls off at the longest wavelengths, so that relatively wide slits were required beyond $2.8 \mu$. However at wavelengths shorter than $2.75 \mu(\nu>3,640$ $\mathrm{cm}^{-1}$ ) the instrument could be operated at its maximum resolving power. Under these conditions records with good signal-to-noise ratios were obtained with spectral slit widths of $0.12 \mathrm{~cm}^{-1}$. This is comparable to, or less than the width of the $\mathrm{H}_{2} \mathrm{O}$ lines, when the water-vapor content of the optical path in the room and the spectrometer was that prevailing on a dry February day. It is often possible to distinguish the presence of two components in lines separated by $0.15 \mathrm{~cm}^{-1}$, and lines $0.30 \mathrm{~cm}^{-1}$ apart are clearly resolved. Further lowering of the watervapor content might result in some further improvement in resolution, but as the present resolution was adequate, and as it was desired to record as many of the weaker lines as possible, this was not attempted.

Figures in brackets indicate the literature references at the end of this paper. 
The source was a tungsten lamp with quartz window, operated at 38-amp current. Radiation of second and higher orders was removed by a tellurium filter. This completely eliminated wavelengths below $1.35 \mu$, so that the spectrum below $2.70 \mu$ was free from higher-order radiation. Above that wavelength the filter reduced but did not completely eliminate the second-order radiation. The resultant overlapping of first- and second-order absorption lines (second-order absorption, due to the $1.4 \mu \mathrm{com}$ bination band of $\mathrm{H}_{2} \mathrm{O}$, is relatively strong) complicates the appearance of the observed spectrum. It does not, however, lead to any difficulty in assigning the first-order lines, which appear broader because of their greater natural width in $\mathrm{cm}^{-1}$; and the presence of the second-order lines is of some value in providing frequent standards for wavelength determinations, as they have been measured with high accuracy [7].

A number of runs through the entire spectral region in question, or through various portions of it, were made at different humidities and with different slit widths. Runs were made with the grating drive operating both toward longer and toward shorter wavelengths, and with both positive and negative values of the grating angle. By intercomparison of these various tracings, it was possible to distinguish weak true absorption lines close to the noise level. In addition, several spectra were obtained with an absorption cell $5 \mathrm{~cm}$ long containing $\mathrm{CO}_{2}$ gas at a pressure of $40 \mathrm{~cm}$ in the optical path, in order to confirm that the lines attributed to $\mathrm{CO}_{2}$ in the atmospheric path were indeed due to that molecule.

The wavelengths of the absorption lines were obtained as follows. The primary standards of wavelength were higher-order lines from a mercury-vapor lamp, which was placed at the center of focus of the spectrometer, so that the mercury lines in emission were superimposed upon the absorption spectrum. This yielded 8 or 10 lines in the region between 2.4 and $2.9 \mu$ whose positions were known with accuracy. The second-order water-vapor lines provided numerous other standards between 2.7 and $2.85 \mu$. A dispersion curve, representing wavelength as a quadratic function of the reading of the Veeder counter, which in turn related position on the recording chart to the grating angle, was passed through the standard lines. This gave the frequency of a number of the first-order absorption lines, namely those close to the standards, with good accuracy. To establish additional standard warelengths in spectral regions further from the primary standards, use was made of the characteristic frequency differences between the lowest rotational levels of the $\mathrm{H}_{2} \mathrm{O}$ molecule. These are known, from their occurrence in a large number of vibrational bands [4] with an accuracy of $\pm 0.02 \mathrm{~cm}^{-1}$, and hence provide a set of reliable internal frequency standards, when the lines in question are not overlapped by other lines. The dispersion curve that was finally adopted was fitted to a number of these secondary standards. The measurement of the positions of the absorption lines, and their reduction to wavelengths, was carried out independently by different observers on several tracings. The final values reported are the averages of these reductions, and are believed accurate to within $\pm 0.20 \mathrm{~cm}^{-1}$. The precision of measurement of the frequency difference between neighboring lines, or of lines close to the primary standards, should be considerably better, about $\pm 0.05 \mathrm{~cm}^{-1}$. The reported frequencies are also subject to a minor uncertainty associated with the conversion factor from wavelengths in air to wave numbers in vacuum. The adopted value was $\nu_{\mathrm{vac}}=\left(\lambda_{\mathrm{air}} \times 1.0002706\right)^{-1}$.

\section{Results}

A reproduction of a tracing made from a recording of the entire region from 4,090 to $3,498 \mathrm{~cm}^{-1}$ is shown in figures 1 to 4 . The tracing is broken into segments approximately $50 \mathrm{~cm}^{-1}$ in length, with an overlap of one or two lines at either end. The wave numbers (in vacuum) of a number of the more prominent lines are indicated in the figure. As explained previously, the spectrum below $3,700 \mathrm{~cm}^{-1}$ is not pure, and hence contains some second-order lines.

The wave numbers and approximate intensities of all the observed lines, together with the identification proposed for most of them, are given in table 1 . The first column of the table presents the intensity, on an estimated scale 0 to 100 , which corresponds roughly to the percentage absorption at the maximum. The intensity scale is of least significance for very intense lines $(I>95)$, of which a few show complete absorption over a width approaching $1 \mathrm{~cm}^{-1}$, for lines that are members of incompletely resolved groups, and for the lines of longest wavelength, where the second-order energy and absorption is a complicating factor. However it is of some quantitative usefulness for well-resolved lines between 10 to 90 in the shorter wavelength region, and serves as a qualitative indication of relative intensity throughout the table. The second column of table 1 gives the vacuum wave number of the line center, or what is judged to be the position of maximum absorption of an incompletely resolved component. 


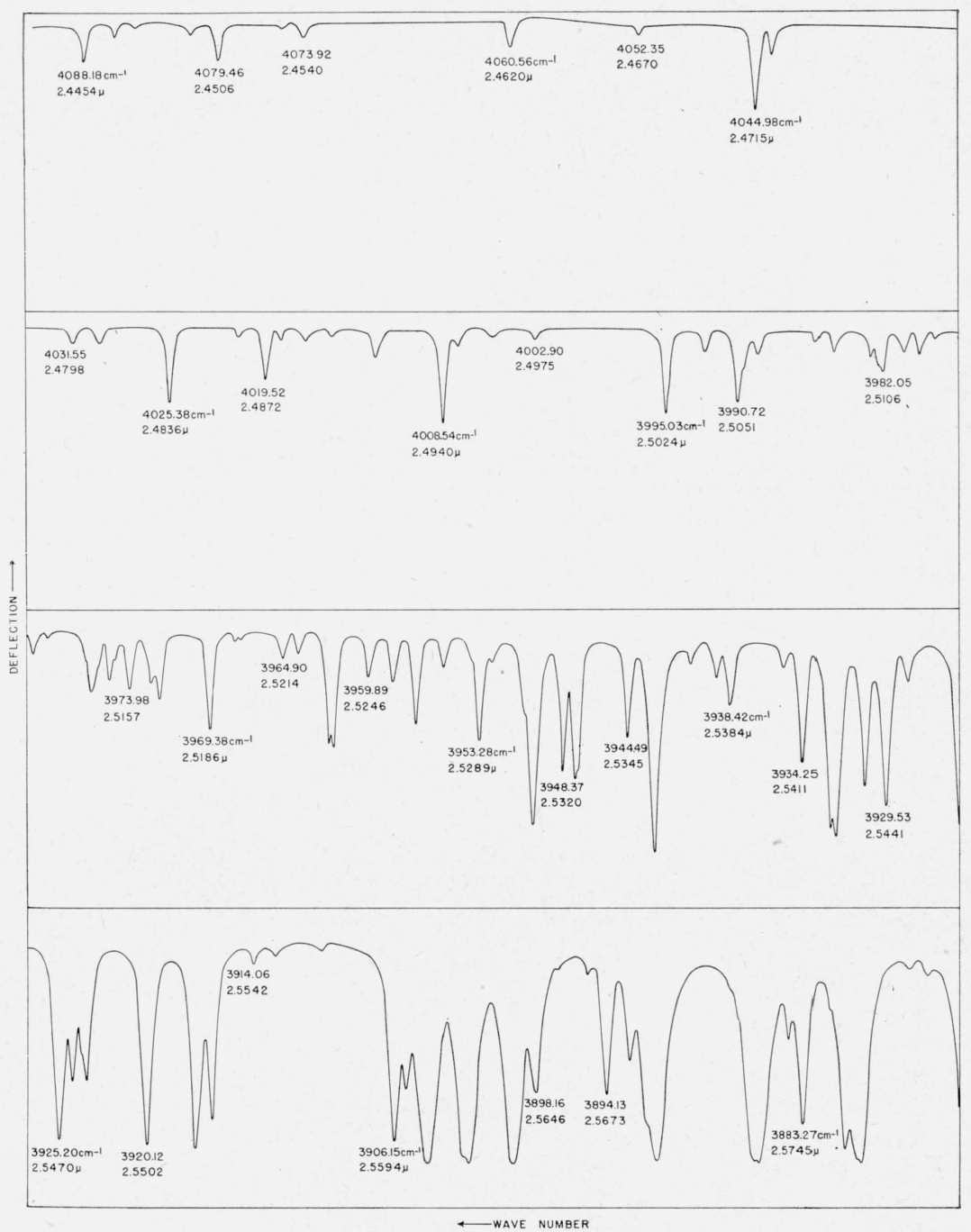

FiguRE 1. Observed absorption due to $\mathrm{H}_{2} \mathrm{O}$ and $\mathrm{CO}_{2}, 4,090$ to $3,875 \mathrm{~cm}^{-1}$. 


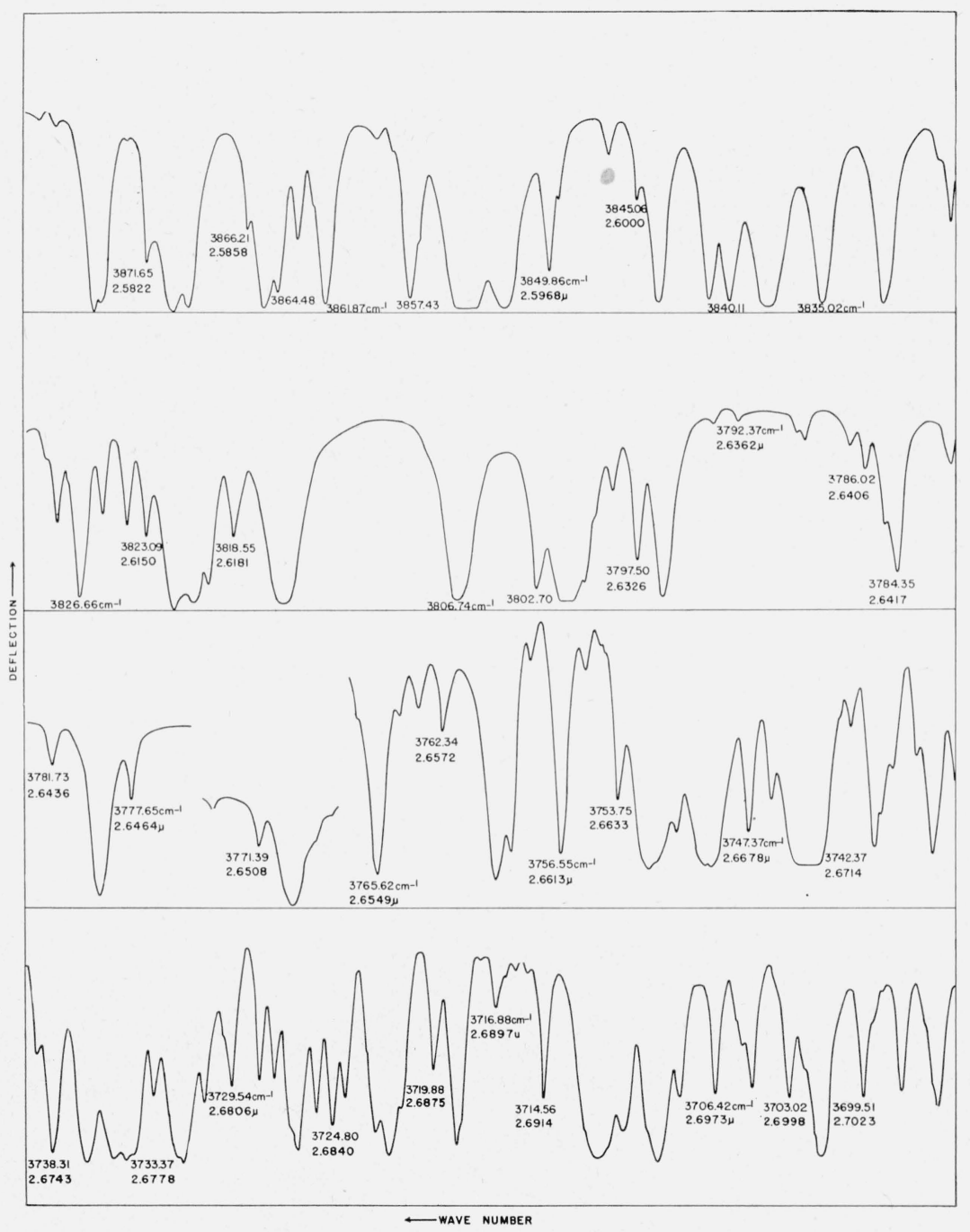

Figure 2. Observed absorption due to $\mathrm{H}_{2} \mathrm{O}$ and $\mathrm{CO}_{2}, 3,877$ to $3,692 \mathrm{~cm}^{-1}$. 


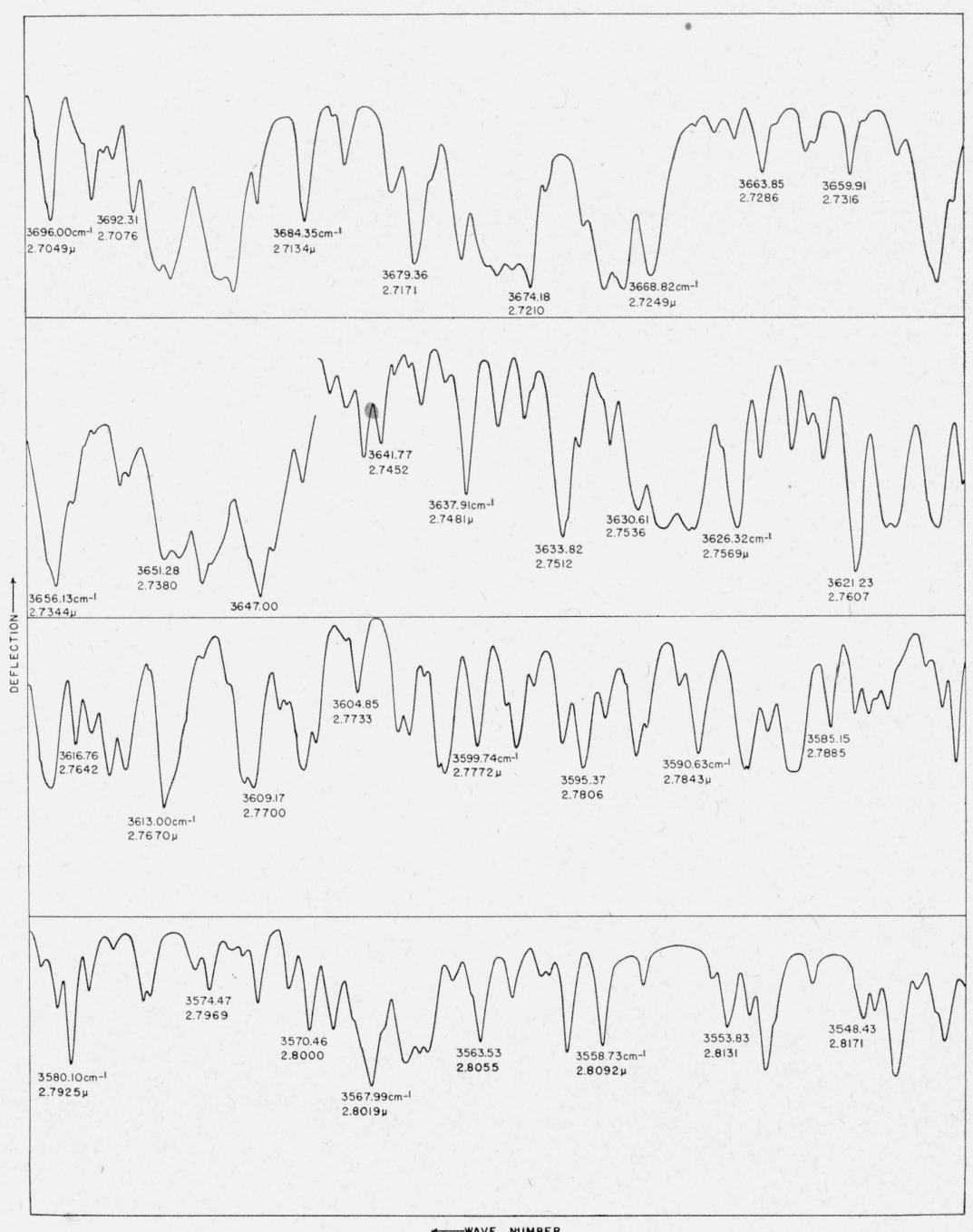

Figure 3. Observed absorption due to $\mathrm{H}_{2} \mathrm{O}$ and $\mathrm{CO}_{2}, 3,697$ to $3,544 \mathrm{~cm}^{-1}$.

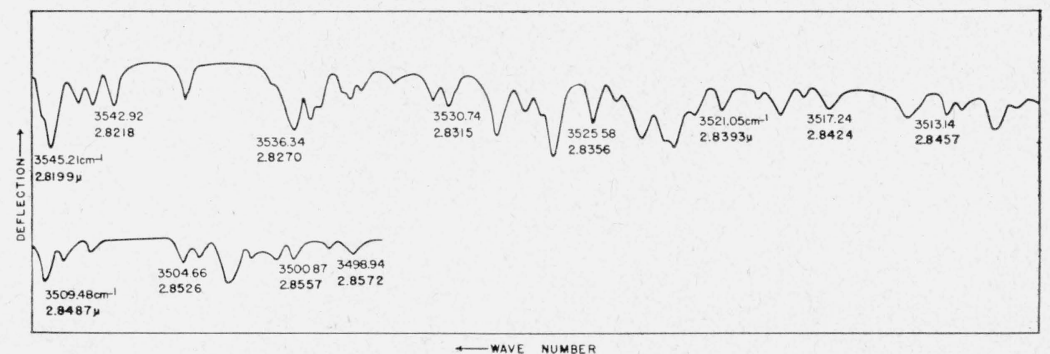

FIgURE 4. Observed absorption due to $\mathrm{H}_{2} \mathrm{O}$ and $\mathrm{CO}_{2}, 3,546$ to $3,498 \mathrm{~cm}^{-1}$. 
TABLE 1. Absorption lines in the region of $2.7 \mu$ due to $\mathrm{H}_{2} \mathrm{O}$ and $\mathrm{CO}_{2}$

\begin{tabular}{|c|c|c|c|c|}
\hline \multicolumn{2}{|c|}{ Observed } & \multirow{2}{*}{$\begin{array}{c}\text { Identification } \\
\qquad J^{\prime} \tau-J^{\prime \prime} \tau\end{array}$} & \multicolumn{2}{|c|}{ Calculated } \\
\hline $\begin{array}{l}\text { Inten- } \\
\text { sity }\end{array}$ & $\nu_{\mathrm{vac}}$ & & $\begin{array}{l}\text { Inten- } \\
\text { sity }\end{array}$ & $\nu$ \\
\hline 10 & $\begin{array}{c}\mathrm{cm}^{-1} \\
3498.94\end{array}$ & & & \\
\hline 5 & 3499. 70 & $8_{2}-9_{1}$ & 0. 066 & 99.60 \\
\hline 12 & 3500.87 & $\begin{array}{l}9_{-5}-10_{-6}^{-6} \\
7001.9 / 2\end{array}$ & $\begin{array}{l}.041 \\
(25)\end{array}$ & $\begin{array}{l}99.95 \\
00.90\end{array}$ \\
\hline 10 & 3501.45 & $\begin{array}{c}8_{0}-9_{-1} \\
10_{-8}-11_{-9}\end{array}$ & $\begin{array}{r}0.143 \\
.080\end{array}$ & $\begin{array}{l}01.41 \\
02.46\end{array}$ \\
\hline 6 & 3502.40 & $\begin{array}{c}10_{-7}-11_{-8} \\
9_{-4}-10_{-5}\end{array}$ & $\begin{array}{r}026 \\
.111 \\
(20)\end{array}$ & $\begin{array}{l}02.58 \\
02.67 \\
03.02\end{array}$ \\
\hline 40 & 3503. 09 & $\begin{array}{c}7006.1 / 2 \\
2_{1}-3_{3}\end{array}$ & 15. $\left.{ }^{(20)}\right)$ & $\begin{array}{l}03.02 \\
03.18\end{array}$ \\
\hline 12 & 3504.08 & $11_{-10}^{-1}-12_{-11}$ & 0.107 & 04.15 \\
\hline 15 & 3504. 66 & $2_{2}-3_{2}$ & 5. $16^{*}$ & 04. 59 \\
\hline 8 & 3507. 87 & $7015.9 / 2$ & $(20)$ & 07. 93 \\
\hline 15 & 3508.80 & $6_{-6}-7_{-6}$ & $\begin{array}{l}\text { 2. } 55^{*} \\
7.67^{*}\end{array}$ & 08. 72 \\
\hline 35 & 3509.48 & $\begin{array}{ll}0_{-5}- & y_{-7} \\
8_{-2}- & 9_{-3}\end{array}$ & 0.232 & $\begin{array}{l}\text { 09. } 41 \\
09.36\end{array}$ \\
\hline 5 & 3510.89 & $\begin{array}{l}8_{-1}-9_{-2} \\
6_{-1}-7_{-3}\end{array}$ & $\begin{array}{l}.053 \\
.647 *\end{array}$ & $\begin{array}{l}\text { 10. } 45 \\
\text { 10. } 75\end{array}$ \\
\hline 25 & 3511.45 & $7022.7 / 2$ & $\begin{array}{r}(30) \\
2.60 *\end{array}$ & $\begin{array}{l}11.35 \\
11.51\end{array}$ \\
\hline 10 & 3512. 61 & $7025.3 / 2$ & $(20)$ & 12. 67 \\
\hline 12 & 3513. 14 & $\begin{array}{cc}3_{-2}- & 4_{1} \\
7026.4 / 2\end{array}$ & $\begin{array}{r}0.081 \\
(20)\end{array}$ & $\begin{array}{l}\text { 12. } 94 \\
\text { 13. } 23\end{array}$ \\
\hline 15 & 3414.47 & $5_{3}-5_{5}$ & $0.61^{*}$ & 14. 36 \\
\hline 10 & 3517. 24 & $7034.4 / 2$ & $\begin{array}{l}(15) \\
0 \quad 052\end{array}$ & 14. 14 \\
\hline 6 & 3518.13 & $7036.4 / 2$ & $\begin{array}{r}0.052 \\
(10)\end{array}$ & $\begin{array}{l}18.44 \\
18.20\end{array}$ \\
\hline 15 & 3518. 92 & $4_{-2}-5_{1}$ & 0. 213 & 18. 90 \\
\hline $\begin{array}{r}5 \\
12\end{array}$ & $\begin{array}{l}3519.57 \\
3521.05\end{array}$ & $\begin{array}{l}7038.4 / 2 \\
7042.1 / 2\end{array}$ & $(30)$ & 21.05 \\
\hline & 3521.05 & $5_{-2}-6_{-4}$ & 1. $45^{*}$ & $\begin{array}{l}21.97 \\
21.97\end{array}$ \\
\hline 15 & 3521.92 & $7044.0 / 2$ & $(30)$ & 22.00 \\
\hline 35 & 3522.69 & $2_{-1}-3_{1}$ & 8. $83^{*}$ & 22. 72 \\
\hline 30 & 3522.99 & $8_{-4}-9_{-5}$ & 0.381 & 23. 07 \\
\hline 25 & 3523.80 & $\begin{array}{l}7047.4 / 2 \\
9-7-10_{-8}\end{array}$ & $\begin{array}{l}(40) \\
0.088\end{array}$ & $\begin{array}{l}23.7 \\
24.00\end{array}$ \\
\hline 8 & 3524.28 & $\begin{array}{l}9-7 \\
9_{-6}-10_{-7}\end{array}$ & .25 & 24. 26 \\
\hline 10 & 3524.69 & $8_{-3}-9_{-4}$ & .110 & 24. 71 \\
\hline 20 & 3525.58 & $\begin{array}{c}10_{-10}-11_{-11} \\
7_{3}-8_{2}\end{array}$ & $\begin{array}{l}337 \\
056\end{array}$ & $\begin{array}{l}25.66 \\
26.92\end{array}$ \\
\hline 40 & 3526.98 & $\begin{array}{c}3-1-4_{-1} \\
7054.1 / 2\end{array}$ & $\begin{array}{c}\text { 11. } 73^{*} \\
(40)\end{array}$ & 27. 03 \\
\hline 18 & 3527.43 & $7_{2}-8_{1}$ & 0.170 & 27. 58 \\
\hline 18 & 3528.07 & $4_{-4}-5_{-1}$ & 0. 261 & 28. 04 \\
\hline 35 & 3528.97 & $5_{-5}-6_{-5}$ & 15. $89^{*}$ & 28. 99 \\
\hline 15 & 3530.74 & $5_{-4}-6_{-6}$ & 4. $48 *$ & 30. 64 \\
\hline 13 & 3531.51 & $7_{1}-8_{0}$ & 0.134 & 31.33 \\
\hline 5 & 3532. 71 & $7065.5 / 2$ & (15) & 32. 76 \\
\hline 8 & 3533.85 & $7067.7 / 2$ & $(20)$ & 33. 82 \\
\hline 12 & 3534.25 & $7068.5 / 2$ & $(20)$ & 34.27 \\
\hline 5 & 3534.55 & $7069.0 / 2$ & (15) & 34.55 \\
\hline 18 & 3535. 27 & $7070.7 / 2$ & $(45)$ & 35. 38 \\
\hline 25 & 3535. 72 & $7071.4 / 2$ & $(60)$ & 35. 73 \\
\hline 32 & 3536. 34 & $\begin{array}{r}7_{0}-8_{-1} \\
7_{-1}-8_{-2}\end{array}$ & $\begin{array}{r}0.436 \\
240\end{array}$ & $\begin{array}{l}36.12 \\
36.50\end{array}$ \\
\hline 10 & 3537. 18 & & & \\
\hline 8 & 3540.23 & $5_{0}-5_{2}$ & $.75^{*}$ & 40. 23 \\
\hline 20 & 3540.38 & $7080.5 / 2$ & $(50)$ & 40. 28 \\
\hline 20 & 3542.92 & $\begin{array}{l}3-1-4_{2} \\
6_{-5}-6_{-3}\end{array}$ & $\begin{array}{l}\text { 0. } 098 \\
\text { 2. } 72^{*}\end{array}$ & $\begin{array}{l}\text { 42. } 89 \\
42.92\end{array}$ \\
\hline 20 & 3543.73 & $\begin{array}{l}6_{6}-7_{5} \\
5_{1}-5_{3} \\
4_{1}-4_{3}\end{array}$ & $\begin{array}{l}\text { 0. } 107 \\
\text { 2. } 31^{*} \\
\text { 2. } 34^{*}\end{array}$ & $\begin{array}{l}43.41 \\
43.66 \\
43.80\end{array}$ \\
\hline 18 & 3544.25 & $2_{0}-3_{0}$ & 5. $49 *$ & 44. 27 \\
\hline
\end{tabular}

TABLE 1. Absorption lines in the region of $2.7 \mu$ due to $\mathrm{H}_{2} \mathrm{O}$ and $\mathrm{CO}_{2}-$ Continued

\begin{tabular}{|c|c|c|c|c|}
\hline \multicolumn{2}{|c|}{ Observed } & \multirow{2}{*}{$\begin{array}{l}\text { Identification } \\
J^{\prime} \tau-J^{\prime \prime} \tau\end{array}$} & \multicolumn{2}{|c|}{ Calculated } \\
\hline $\begin{array}{l}\text { Inten- } \\
\text { sity }\end{array}$ & $\nu_{\mathrm{vac}}$ & & $\begin{array}{l}\text { Inten- } \\
\text { sity }\end{array}$ & $\nu$ \\
\hline & $\mathrm{cm}^{-1}$ & & & \\
\hline 45 & 3545. 21 & $\begin{array}{l}7_{-3}-8_{-4} \\
8_{-6}-9_{-}\end{array}$ & $\begin{array}{r}0.36 \\
75\end{array}$ & $\begin{array}{l}\text { 44. } 88 \\
45.19\end{array}$ \\
\hline 25 & 3545.56 & $8_{-5}^{-6}-9_{-6}$ & 0.24 & 45. 35 \\
\hline 12 & 3546. 28 & $7092.5 / 2$ & (35) & 46. 23 \\
\hline & & $9_{-8}-10_{-9}$ & 0.97 & 46. 86 \\
\hline 6.5 & 3046.15 & $\begin{array}{c}7_{-2}-8_{-3} \\
7094.7 / 2\end{array}$ & $\begin{array}{l}91 \\
(60)\end{array}$ & $\begin{array}{l}\text { 47. } 03 \\
47.35\end{array}$ \\
\hline 23 & 354705 & $7095.9 / 2$ & $(55)$ & 47. 92 \\
\hline 20 & 50+7. & $2_{-1}-3_{2}$ & 0.023 & 48. 11 \\
\hline 25 & 3548.43 & $4_{-4}-55_{-4}$ & 6. $84^{*}$ & 48. 43 \\
\hline 10 & 3548.74 & $7097.4 / 2$ & $2^{(30)}$ & $\begin{array}{l}48.75 \\
50.33\end{array}$ \\
\hline 15 & 3550.42 & $\begin{array}{c}{ }_{-3}-0_{-1} \\
6_{0}-6^{2}\end{array}$ & $\begin{array}{l}\text { 2. } \\
0.52^{*}\end{array}$ & $\begin{array}{l}50.33 \\
50.35\end{array}$ \\
\hline 20 & 3551.96 & $4_{-1}-5_{-3}$ & 5. $73^{*}$ & 51. 76 \\
\hline & & $6_{1}-7_{-1}$ & $0.17 * *$ & 52. 12 \\
\hline 65 & 3552. 29 & $\begin{array}{l}4_{-3}-5_{-5} \\
7104.7 / 2\end{array}$ & $\begin{array}{r}20.39^{*} \\
(55)\end{array}$ & $\begin{array}{l}52.24 \\
52.30\end{array}$ \\
\hline & & $6_{-6}-6_{-4}$ & $0.99^{*}$ & 52. 28 \\
\hline 28 & 3552.90 & $7105.9 / 2$ & $(60)$ & 52. 94 \\
\hline 35 & 3553. 83 & $6_{4}-7_{3}$ & 0.57 & 53. 83 \\
\hline 12 & 3554.36 & $7108.6 / 2$ & $(30)$ & 54. 33 \\
\hline 15 & 3557. 12 & $1_{0}-2_{2}$ & 5. $98^{*}$ & 57. 11 \\
\hline 50 & 3558. 73 & $7117.6 / 2$ & $(90)$ & 58. 72 \\
\hline 55 & 3560. 12 & $\begin{array}{cc}6_{2}- & 7_{1} \\
7120.3 / 2\end{array}$ & $\begin{array}{r}0.94 \\
(35)\end{array}$ & $\begin{array}{l}60.12 \\
60.20\end{array}$ \\
\hline & & $5_{-2}-5_{0}$ & 1. $72 *$ & 60.84 \\
\hline 10 & 3560.86 & $7120.3 / 2$ & $(25)$ & 60.88 \\
\hline & & $7_{-1}-7_{1}$ & $0.84^{*}$ & 60. 91 \\
\hline 12 & 3561. 12 & $5_{-4}-5_{-2}$ & 1. $82 *$ & 61. 23 \\
\hline 25 & 3562.33 & $6_{1}-7_{0}$ & 0.32 & 62.30 \\
\hline 50 & 3563.53 & $1_{1}-2_{1}$ & 21. $8^{*}$ & 63.53 \\
\hline 25 & 3563. 89 & $7_{-5}-7_{-3}$ & 1. $87 *$ & 64.01 \\
\hline 10 & 3564.67 & (a) & & \\
\hline 60 & 3565.69 & $6_{0}-7_{-1}$ & 1. 85 & $\begin{array}{l}65.64 \\
65.98\end{array}$ \\
\hline 60 & 3566.02 & $\begin{array}{r}7_{-5}-8_{-6} \\
7_{1}-8_{-3}\end{array}$ & $\begin{array}{l}0.57 \\
.05 * *\end{array}$ & $\begin{array}{l}65.98 \\
66.23\end{array}$ \\
\hline & & $3-3-4_{-3}$ & $25.8^{*}$ & 66.52 \\
\hline 65 & 3500.51 & $7_{-4}-8_{-5}$ & 1. 85 & 66.82 \\
\hline 40 & 3567.47 & $7134.9 / 2$ & (55) & 67. 42 \\
\hline 80 & 3567. 99 & $\begin{array}{c}8_{-8}-9_{-9} \\
7136.0 / 2\end{array}$ & $\begin{array}{l}2.46 \\
(40)\end{array}$ & $\begin{array}{l}68.00 \\
68.04\end{array}$ \\
\hline 40 & 3568. 35 & $6_{-2}-7_{-3}$ & 2. 84 & 68. 33 \\
\hline 30 & 3568. 69 & $4_{-1}-4_{1}$ & 7. 07 & 68.72 \\
\hline 45 & 3569. 48 & $7139.0 / 2$ & $(80)$ & 69.52 \\
\hline 47 & 3570.46 & $6_{-1}-7_{-2}$ & 0.70 & 70.53 \\
\hline 25 & 3571.27 & $7142.6 / 2$ & $(65)$ & 71. 30 \\
\hline 32 & 3572.55 & $7145.1 / 2$ & $(80)$ & 72.57 \\
\hline 10 & 3573. 16 & $7_{3}-8_{-1}$ & $0.31 * *$ & 73. 09 \\
\hline 8 & 3573.59 & $3_{0}-3_{2}$ & 2. $23^{*}$ & 73.57 \\
\hline 25 & 3574.47 & $3_{-2}-4_{-4}$ & 9. $95^{*}$ & 74. 47 \\
\hline 15 & 3575.10 & $3_{-3}-4_{0}$ & 0. 193 & 75. 07 \\
\hline 30 & 3576.88 & $5_{-5}-5_{-3}$ & 6. $59^{*}$ & 76. 78 \\
\hline 35 & 3577. 17 & $4_{-3}-4_{-1}$ & 9. $31^{*}$ & 77. 22 \\
\hline 15 & 3577. 40 & $\mathrm{CO}_{2} \quad \mathrm{P}_{40}$ & & 77. 38 \\
\hline 8 & 3578.38 & $7156.7 / 2$ & $(35)$ & 78. 38 \\
\hline 25 & 3579.39 & $\begin{array}{r}3_{1}-3_{3} \\
C\end{array}$ & 7. $04^{*}$ & $\begin{array}{l}\text { 79. } 17 \\
79.36\end{array}$ \\
\hline & & $5_{4}-6_{3}$ & 0.61 & 79.87 \\
\hline 70 & 3580.10 & $7160.2 / 2$ & $(65)$ & 80.08 \\
\hline 40 & 3580.66 & $7161.3 / 2$ & $(75)$ & 80.66 \\
\hline 20 & 3581.33 & $\mathrm{CO}_{2} \quad \mathrm{P}_{36}$ & & 81.33 \\
\hline 15 & 3582.44 & $9_{-8}-9_{-7}$ & 0.040 & 82. 41 \\
\hline 40 & 3582.95 & $7165.7 / 2$ & $(65)$ & 82. 84 \\
\hline 20 & 3583. 33 & $\begin{array}{r}U_{2}-{ }_{34} \\
3_{0}-4_{-2}\end{array}$ & 1. $89^{*}$ & $\begin{array}{l}\text { 80. } 20 \\
83.38\end{array}$ \\
\hline
\end{tabular}

a This line very probably is $5_{5}-43$ in the overtone band $2 \nu_{2}$, the strongest expected line of that band in this region. 
TABLE 1. Absorption lines in the region of $2.7 \mu$ due to $\mathrm{H}_{2} \mathrm{O}$ and $\mathrm{CO}_{2}-$ Continued

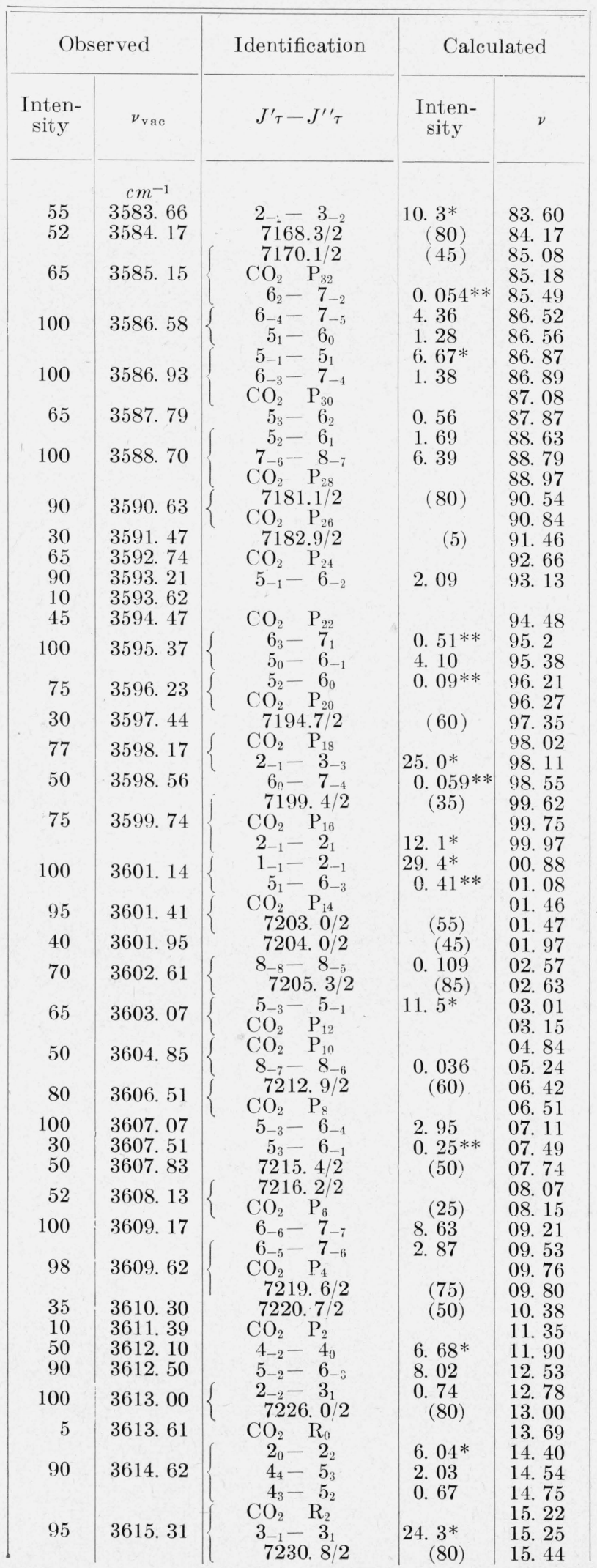

TABLE 1. Absorption lines in the region of $2.7 \mu$ due to $\mathrm{H}_{2} \mathrm{O}$ and $\mathrm{CO}_{2}$-Continued

\begin{tabular}{|c|c|c|c|c|}
\hline \multicolumn{2}{|c|}{ Observed } & \multirow{2}{*}{$\begin{array}{c}\text { Identification } \\
\qquad J^{\prime} \tau-J^{\prime \prime} \tau\end{array}$} & \multicolumn{2}{|c|}{ Calculated } \\
\hline $\begin{array}{l}\text { Inten- } \\
\text { sity }\end{array}$ & $\nu_{\mathrm{vac}}$ & & $\begin{array}{l}\text { Inten- } \\
\text { sity }\end{array}$ & $\nu$ \\
\hline & $\mathrm{cm}^{-1}$ & & & \\
\hline 60 & 3616.08 & 7231. $9 / 2$ & $(90)$ & 16. 14 \\
\hline 65 & 3616.76 & $\begin{array}{c}7233.2 / 2 \\
\mathrm{CO}_{2} \mathrm{R}_{4}\end{array}$ & $(80)$ & $\begin{array}{l}\text { 16. } 70 \\
\text { 16. } 73\end{array}$ \\
\hline 100 & 3617. 82 & $4_{2}-5_{1}$ & 6. 22 & 17. 83 \\
\hline 98 & 3618.18 & $\begin{aligned} 3_{-3}- & 3_{-1} \\
8_{-6}- & 8_{-3} \\
\mathrm{CO}_{2} & \mathrm{R}_{6}\end{aligned}$ & $\begin{array}{r}25.7^{*} \\
0.080\end{array}$ & $\begin{array}{l}\text { 17. } 99 \\
18.18 \\
18.22\end{array}$ \\
\hline $100 w$ & 3619.81 & $\begin{aligned} 4_{0} & -5-1 \\
\mathrm{CO}_{2} & \mathrm{R}_{8} \\
0 & -1_{0}\end{aligned}$ & $\begin{array}{l}11.5 \\
3.33^{*}\end{array}$ & $\begin{array}{l}19.66 \\
19.69 \\
19.92\end{array}$ \\
\hline 100 & 3621.23 & $\begin{array}{c}\mathrm{CO}_{2} \quad \mathrm{R}_{10} \\
4_{1}-5_{0} \\
7242.6 / 2\end{array}$ & $\begin{array}{l}2.13 \\
(80)\end{array}$ & $\begin{array}{l}\text { 21. } 14 \\
21.19 \\
21.32\end{array}$ \\
\hline 45 & 3622.59 & $\begin{array}{ll}\mathrm{CO}_{2} & \mathrm{R}_{12}\end{array}$ & $(00)$ & $\begin{array}{l}21.02 \\
22.57\end{array}$ \\
\hline 15 & 3622.82 & $\begin{array}{c}7245.5 / 2 \\
10-2\end{array}$ & $\begin{array}{r}(55) \\
5.18^{*}\end{array}$ & $\begin{array}{l}22.76 \\
23.20\end{array}$ \\
\hline 25 & 3623. 25 & $7_{-7}-7_{-4}$ & 0.09 & 23. 29 \\
\hline 40 & $\begin{array}{l}3623.95 \\
3625\end{array}$ & $\mathrm{CO}_{2}$ & & 23. 96 \\
\hline 45 & 3625.33 & $\mathrm{CO}_{2} \quad \mathrm{R}_{16}$ & & 25. 33 \\
\hline 99 & 3626.32 & $\begin{array}{c}4_{3}-5_{1} \\
7252.7 / 2\end{array}$ & $\begin{array}{l}0.23 * * \\
(55)\end{array}$ & $\begin{array}{l}26.37 \\
26.35\end{array}$ \\
\hline 75 & 3626. 61 & $\begin{array}{ll}\mathrm{CO}_{2} & \mathrm{R}_{18}\end{array}$ & & 26. 67 \\
\hline 20 & 3626.94 & $7253.9 / 2$ & $(50)$ & 26. 97 \\
\hline 100 & 3628.14 & $\mathrm{CO}_{2}-\mathrm{R}_{20}$ & & 28. 00 \\
\hline 100 & 3628.42 & $4_{-2}-5_{-3}$ & 16. 25 & 28. 24 \\
\hline 99 & 3629.35 & $\mathrm{CO}_{2}-\mathrm{R}_{22}$ & 5. 21 & $\begin{array}{l}29.30 \\
29.35\end{array}$ \\
\hline 100 & 3629.65 & $\begin{array}{rr}5_{-4} & -6_{-5}^{-5} \\
\mathrm{CO}_{2} & \mathrm{R}_{24}\end{array}$ & 15. 6 & $\begin{array}{l}29.62 \\
30.59\end{array}$ \\
\hline 85 & 3630.61 & $\begin{array}{c}4_{4}-5_{0} \\
2_{-2}-2_{0} \\
7262.3 / 2\end{array}$ & $\begin{array}{l}0.075 * * \\
12.4^{*} \\
(75)\end{array}$ & $\begin{array}{l}\text { 30. } 69 \\
\text { 30. } 70 \\
\text { 31. } 12\end{array}$ \\
\hline 35 & 3631.80 & $\mathrm{CO}_{2} \quad \mathrm{R}_{26}$ & & 31.86 \\
\hline 10 & 3632. 17 & $7_{-5}-7_{-2}$ & 0.057 & 32. 30 \\
\hline $\begin{array}{l}35 \\
20\end{array}$ & $\begin{array}{l}3633.09 \\
3633.64\end{array}$ & $\mathrm{CO}_{2} \quad \mathrm{R}_{28}$ & & 33.10 \\
\hline 98 & 3633.82 & $\begin{array}{l}4_{-1}-5_{-2} \\
7267.9 / 2\end{array}$ & $\begin{array}{l}\text { 4. } 39 \\
(50)\end{array}$ & $\begin{array}{l}33.82 \\
33.94\end{array}$ \\
\hline 50 & 3634. 40 & $\mathrm{CO}_{2} \quad \mathrm{R}_{30}$ & & 34. 33 \\
\hline 10 & 3635. 14 & $3_{3}-4_{-1}$ & $0.78 * *$ & 34. 79 \\
\hline 30 & 3635. 48 & $\mathrm{CO}_{2} \quad \mathrm{R}_{32}$ & & 35.52 \\
\hline 35 & 3636.57 & $\mathrm{CO}_{2}$ & & 36. 69 \\
\hline 70 & 3637. 99 & $\mathrm{CO}_{2} \quad \mathrm{R}_{36}$ & & $\begin{array}{l}37.83 \\
37\end{array}$ \\
\hline 10 & 3638. 88 & $\begin{array}{ll}\mathrm{CO}_{2} & \mathrm{R}_{38}\end{array}$ & J0. & $\begin{array}{l}38.99 \\
38.95\end{array}$ \\
\hline & & $3_{1}-4_{-3}$ & 1. $09 * *$ & 39. 74 \\
\hline 18 & 3639.98 & $\begin{aligned} 4_{0}- & 5_{-4} \\
\mathrm{CO}_{2} & \mathrm{R}_{40}\end{aligned}$ & $0.19 * *$ & $\begin{array}{l}39.88 \\
40.06\end{array}$ \\
\hline 5 & 3640.47 & $7281.1 / 2$ & (40) & 40.53 \\
\hline 6 & 3641.11 & $\mathrm{CO}_{2} \quad \mathrm{R}_{42}$ & & 41. 13 \\
\hline 50 & 3641.77 & $\begin{array}{l}3_{-1}-3_{2} \\
6_{-4}-6_{-1}\end{array}$ & $\begin{array}{l}0.037 \\
.28\end{array}$ & $\begin{array}{l}41.50 \\
41.75\end{array}$ \\
\hline 55 & 3642.53 & $6_{-6}-6_{-3}$ & $\begin{array}{l}.59 \\
038\end{array}$ & 42. 57 \\
\hline & & $\begin{array}{l}8_{-5}-8_{-4} \\
1_{-1}-2_{2}\end{array}$ & $\begin{array}{l}038 \\
.14\end{array}$ & 42. 76 \\
\hline $20 w$ & 3643. 30 & $7287.0 / 2$ & $(60)$ & 43.52 \\
\hline 15 & 3643. 93 & $7288.1 / 2$ & (65) & 44. 02 \\
\hline 30 & 3645.12 & $4_{-2}-4_{1}$ & 0.24 & 45. 20 \\
\hline 90 & 3646.41 & $\begin{array}{r}5_{-3}-5_{9} \\
3_{3}-4_{2}\end{array}$ & $\begin{array}{l}\text { 2. } 106 \\
\text { 2. } 23\end{array}$ & $\begin{array}{l}\text { 45. } 98 \\
\text { 46. } 41\end{array}$ \\
\hline 100 & 3647.00 & $\begin{array}{l}7294.1 / 2 \\
31-4\end{array}$ & $(95)$ & 47. 03 \\
\hline 95 & 3647. 48 & $3_{2}-4_{1}$ & 6. 71 & 47. 53 \\
\hline 100 & 3649. 19 & $4_{-4}-5_{-5}$ & 24. 9 & 49. 18 \\
\hline 100 & 3649.67 & $7299.3 / 2$ & $(85)$ & 49. 67 \\
\hline 98 & 3650.63 & $4_{-3}-5_{-4}$ & 8. 13 & 50.68 \\
\hline 99 & 3651.28 & $3_{-1}-4_{-2}$ & 8. 38 & 51. 27 \\
\hline
\end{tabular}


TABLE 1. Absorption lines in the region of $2.7 \mu$ due to $\mathrm{H}_{2} \mathrm{O}$ and $\mathrm{CO}_{2}-$ Continued

\begin{tabular}{|c|c|c|c|c|}
\hline \multicolumn{2}{|c|}{ Observed } & \multirow{2}{*}{$\begin{array}{l}\text { Identification } \\
\qquad J^{\prime} \tau-J^{\prime \prime} \tau\end{array}$} & \multicolumn{2}{|c|}{ Calculated } \\
\hline $\begin{array}{l}\text { Inten- } \\
\text { sity }\end{array}$ & $\nu_{\mathrm{vac}}$ & & $\begin{array}{l}\text { Inten- } \\
\text { sity }\end{array}$ & $\nu$ \\
\hline & $\mathrm{cm}^{-1}$ & & & \\
\hline 20 & 3653. 05 & $6_{-5}-6_{-4}$ & 0. 23 & 53. 09 \\
\hline $\begin{array}{r}22 \\
5\end{array}$ & $\begin{array}{l}3653.32 \\
3654\end{array}$ & $7306.6 / 2$ & $(95)$ & 53.33 \\
\hline 10 & $\begin{array}{l}5004.4 \\
3654.91\end{array}$ & & & \\
\hline 30 & 3655.35 & $7310.7 / 2$ & $(85)$ & 55. 32 \\
\hline 100 & 3656. 13 & $7312.2 / 2$ & $(70)$ & 56.07 \\
\hline 85 & 3656.46 & $3_{0}-4_{-1}$ & 17. 7 & 56. 22 \\
\hline 15 & 3657.88 & $7315.7 / 2$ & $(70)$ & 57. 83 \\
\hline 5 & 3659.25 & $7318.6 / 2$ & $(25)$ & 59. 37 \\
\hline 30 & 3659. 91 & $5_{-5}-5_{-2}$ & 0.35 & 59. 94 \\
\hline 15 & 3661.51 & $7322.8 / 2$ & $(60)$ & 61. 43 \\
\hline 18 & 3661.87 & $7323.8 / 2$ & $(60)$ & 61. 93 \\
\hline 10 & 3662.05 & & & \\
\hline 10 & 3661. 22 & & & \\
\hline 30 & 3663.85 & $7327.7 / 2$ & $(90)$ & 63. 91 \\
\hline 15 & 3665. 08 & $7330.2 / 2$ & $(60)$ & 65. 11 \\
\hline 10 & 3666. 00 & $9_{-4}-9_{-3}$ & 0.036 & 65. 92 \\
\hline 3 & 3666. 90 & $7333.8 / 2$ & (15) & 66. 92 \\
\hline 99 & 3668. 82 & $3_{-3}-4_{-4}$ & 11. 6 & 68. 76 \\
\hline 100 & 3670.03 & $7339.9 / 2$ & (90) & 70. 00 \\
\hline 95 & 3670.41 & $7_{-4}-7_{-3}$ & 0.37 & 70.05 \\
\hline 100 & 3670.92 & $3_{-2}-4_{-3}$ & 33. 2 & 70. 60 \\
\hline 40 & 3671.83 & $7343.7 / 2$ & $(85)$ & 71. 89 \\
\hline 30 & 3673.56 & $7347.0 / 2$ & $(65)$ & 73. 52 \\
\hline 100 & 3674.18 & $4_{-4}-4_{-1}$ & 1. 51 & 74. 16 \\
\hline & & & ${ }_{20}^{(80)}$ & 74. 20 \\
\hline 98 & 3674.95 & $\begin{array}{l}1_{1}-1_{-1} \\
2_{2}-3_{1}\end{array}$ & $\begin{array}{l}39.3^{*} \\
12.5\end{array}$ & $\begin{array}{l}74.65 \\
74.98\end{array}$ \\
\hline 100 & 3675.72 & $7351.5 / 2$ & (35) & 75. 72 \\
\hline 95 & 3676.07 & $2_{0}-3_{-1}$ & 43.7 & 75. 77 \\
\hline 90 & 3677. 30 & $5_{-4}-5_{-3}$ & 1. 53 & 77. 41 \\
\hline 30 & 3678.67 & $\mathrm{CO}_{2} \quad \mathrm{P}_{40}$ & & 78. 54 \\
\hline 92 & 3679.36 & $2_{1}-3_{0}$ & 5. 49 & 79. 36 \\
\hline 50 & 3680.45 & $7361.5 / 2$ & 14. $(80)$ & 80.70 \\
\hline & & $\mathrm{CO}_{2} \mathrm{P}_{38}$ & & $\begin{array}{l}80.58 \\
82.54\end{array}$ \\
\hline 30 & 3682.57 & $\begin{aligned} & 7365.1 / 2 \\
\mathrm{CO}_{2} & \mathrm{P}_{36}\end{aligned}$ & $(50)$ & $\begin{array}{l}82.54 \\
82.61\end{array}$ \\
\hline 5 & 3683. 05 & & & \\
\hline 65 & 3684. 35 & $\begin{array}{l}7368.5 / 2 \\
7368.9 / 2\end{array}$ & $\begin{array}{l}(80) \\
(50)\end{array}$ & $\begin{array}{l}\text { 84. } 17 \\
84.41\end{array}$ \\
\hline 30 & 3684.53 & $3_{-3}-3_{0}$ & 0.48 & $\begin{array}{l}84.52 \\
84.58\end{array}$ \\
\hline 55 & 3686.54 & $\begin{array}{ll}\mathrm{CO}_{2} & \mathrm{P}_{32}\end{array}$ & & 86.54 \\
\hline 100 & 3687. 69 & $7375.7 / 2$ & $(90)$ & 87. 76 \\
\hline $100 \mathrm{w}$ & 3688. 28 & $2_{-2}-3_{-3}$ & 37. 1 & 88. 17 \\
\hline & & $\begin{array}{ll}\mathrm{CO}_{2} & \mathrm{P}_{28}\end{array}$ & & 90.40 \\
\hline 98 & 3690. & $7381.6 / 2$ & $(75)$ & 90.80 \\
\hline 96 & 3691. 08 & $\begin{array}{r}2_{-1}-3_{-2} \\
3_{1}-3_{-1}\end{array}$ & $\begin{array}{l}\text { 12. } 3 \\
30.0 *\end{array}$ & $\begin{array}{l}91.09 \\
91.21\end{array}$ \\
\hline 60 & 3692.31 & $\begin{array}{ll}\mathrm{CO}_{2} & \mathrm{P}_{26}-1\end{array}$ & & 92. 29 \\
\hline 25 & 3693. 20 & $\begin{array}{ll}1 & 0\end{array}$ & 10. 0 * & 93. 26 \\
\hline 18 & 3693. 66 & $6_{-3}-6_{-2}$ & 0.37 & 93. 72 \\
\hline 50 & 3694. 14 & $\mathrm{CO}_{2} \quad \mathrm{P}_{24}$ & & 94. 16 \\
\hline 15 & 3694. 60 & $7390.2 / 2$ & $(60)$ & 95. 06 \\
\hline 60 & 3696. 00 & $\mathrm{CO}_{2} \quad \mathrm{P}_{22}$ & & 96.01 \\
\hline 40 & 3696. 22 & $5_{-1}-5_{-3}$ & 8. $76^{*}$ & 96.21 \\
\hline 20 & 3696. 72 & $7393.9 / 2$ & $(50)$ & 96.95 \\
\hline 52 & 3697. 73 & $\mathrm{CO}_{2} \quad \mathrm{P}_{20}^{\prime}$ & & 97.83 \\
\hline 3 & 3698. 56 & & & \\
\hline 15 & 3699.08 & $\begin{array}{r}7397.7 / 2 \\
\mathrm{p}\end{array}$ & $(40)$ & $\begin{array}{l}98.84 \\
00.50\end{array}$ \\
\hline 50 & 3699.51 & $\begin{array}{r}\mathrm{CO}_{2} \\
7_{6}-\mathrm{P}_{18} \\
7_{7}\end{array}$ & 0.30 & $\begin{array}{l}99.59 \\
01.28\end{array}$ \\
\hline 98 & 3701.45 & $\mathrm{CO}_{2} \quad \mathrm{P}_{16}$ & & 01. 35 \\
\hline 80 & 3701.80 & $\begin{array}{l}1_{1}-2_{0} \\
4_{-3}-4_{-2}\end{array}$ & 25. 10 & $\begin{array}{l}01.79 \\
01.79\end{array}$ \\
\hline
\end{tabular}

TABLE 1. Absorption lines in the region of $2.7 \mu$ due to $\mathrm{H}_{2} \mathrm{O}$ and $\mathrm{CO}_{2}$ - Continued

\begin{tabular}{|c|c|c|c|c|}
\hline \multicolumn{2}{|c|}{ Observed } & \multirow{2}{*}{$\begin{array}{l}\text { Identification } \\
\qquad J^{\prime} \tau-J^{\prime \prime} \tau\end{array}$} & \multicolumn{2}{|c|}{ Calculated } \\
\hline $\begin{array}{l}\text { Inten- } \\
\text { sity }\end{array}$ & $\nu_{\mathrm{vac}}$ & & $\begin{array}{c}\text { Inten- } \\
\text { sity }\end{array}$ & $\nu$ \\
\hline & $\mathrm{cm}^{-1}$ & & & \\
\hline 40 & 3702.15 & $7405.1 / 2$ & $(45)$ & 02.56 \\
\hline 50 & 3703. 02 & $\mathrm{CO}_{2} \quad \mathrm{P}_{14}$ & & 03. 08 \\
\hline 45 & 3704.74 & $\mathrm{CO}_{2} \quad \mathrm{P}_{12}$ & & 04. 78 \\
\hline 30 & 3705. 24 & $\begin{array}{l}5_{1}-6_{-4} \\
6_{0}-6_{-2}\end{array}$ & $\begin{array}{l}0.025 \\
1.89 * *\end{array}$ & $\begin{array}{l}05.23 \\
05.38\end{array}$ \\
\hline 15 & 3705. 44 & $4_{-2}-4_{-4}$ & 6. $14^{*}$ & 05. 59 \\
\hline 48 & 3706.42 & $\mathrm{CO}_{2} \quad \mathrm{P}_{10}$ & & 06. 49 \\
\hline 50 & 3708.08 & $\begin{aligned} 5_{1}- & 5_{-1} \\
\mathrm{CO}_{2} & \mathrm{P}_{8}\end{aligned}$ & $9.34^{*}$ & $\begin{array}{l}07.50 \\
08.07\end{array}$ \\
\hline 99 & 3709.16 & $2_{1}-2_{-1}$ & 16. $3^{*}$ & 09. 10 \\
\hline 93 & 1709. 10 & $1_{-1}-2_{-2}$ & 13.5 & $\begin{array}{l}09.30 \\
09.82\end{array}$ \\
\hline 80 & $\begin{array}{l}3709.71 \\
3710.80\end{array}$ & $\mathrm{CO}_{2}, \mathrm{P}_{6}$ & 0.60 & $\begin{array}{l}09.82 \\
10.82\end{array}$ \\
\hline 80 & 3710.80 & $\begin{array}{l}2_{-2}-1_{-1} \\
2_{-1}\end{array}$ & 39. $6^{*}$ & 11. 09 \\
\hline 95 & 3711.44 & $\begin{array}{ll}\mathrm{CO}_{2} & \mathrm{P}_{4}\end{array}$ & & 11. 44 \\
\hline 100 & 3712.01 & $3_{-3}-2_{-1}$ & 34. $1^{*}$ & 11. 87 \\
\hline & & $\begin{array}{l}10-1 \\
7_{4}-7_{5}\end{array}$ & $\begin{array}{r}29.5 \\
0.55\end{array}$ & 12. 64 \\
\hline 90 & 3712.60 & $\mathrm{CO}_{2} \quad \mathrm{P}_{2}$ & & 13. 03 \\
\hline 70 & 3714.56 & $6_{6}-6_{5}$ & 1. 59 & 14. 65 \\
\hline 5 & 3715.41 & $\mathrm{CO}_{2} \quad \mathrm{R}_{0}$ & & 15. 37 \\
\hline 5 & 3715.94 & $\begin{array}{l}4_{2}-4_{0} \\
0\end{array}$ & 4. $29^{*}$ & 16. 02 \\
\hline 5 & 3716.50 & $\begin{array}{l}y_{0}-9_{1} \\
3_{0}-3_{-2}\end{array}$ & $\begin{array}{l}0.052 \\
6.03 *\end{array}$ & $\begin{array}{l}10.03 \\
16.55\end{array}$ \\
\hline 20 & 3716.88 & $\begin{array}{ll}\mathrm{CO}_{2} & \mathrm{R}_{2}\end{array}$ & & 16. 90 \\
\hline 3 & 3717.68 & & & \\
\hline 60 & 3718. 49 & $\begin{array}{ll}\mathrm{CO}_{2} & \mathrm{R}_{4}\end{array}$ & & 18. 41 \\
\hline 95 & 3718.80 & $5_{-2}-5_{-1}$ & 3. 17 & 18. 95 \\
\hline 50 & 3719.88 & $\mathrm{CO}_{2} \quad \mathrm{R}_{6}$ & & 19. 83 \\
\hline 80 & 3821.42 & $\mathrm{CO}_{2} \quad \mathrm{R}_{8}$ & & 21. 35 \\
\hline 100 & 3722.03 & $\begin{aligned} 8_{2}- & 8_{1} \\
3 & -3_{-1}\end{aligned}$ & $\begin{array}{l}0.19 \\
7.01\end{array}$ & $\begin{array}{l}21.87 \\
22.07\end{array}$ \\
\hline 90 & 3722.64 & $\mathrm{CO}_{2}^{-2} \quad \mathrm{R}_{10}^{-1}$ & & 22. 79 \\
\hline & & $7_{2}-7_{3}$ & 0.64 & 22. 88 \\
\hline 50 & 3723.06 & $\begin{array}{ll}3_{3} & 3_{1}\end{array}$ & 10. $45^{*}$ & 23. 01 \\
\hline & & $7_{3}-7_{2}$ & 0. 21 * & $\begin{array}{l}23.22 \\
24.15\end{array}$ \\
\hline 70 & 3724.11 & 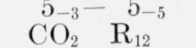 & $10.74^{*}$ & $\begin{array}{l}24.15 \\
24.20\end{array}$ \\
\hline 85 & 3724.80 & $6_{4}-6_{3}$ & 2. 55 & 25. 03 \\
\hline 80 & 3725. 49 & $\mathrm{CO}_{2} \quad \mathrm{R}_{14}$ & 0.68 & 25. 58 \\
\hline 50 & 3726.00 & $\begin{array}{l}0-1-4_{-3}^{0} \\
4_{-1}-4_{-3}\end{array}$ & 14. 0 * & 26. 38 \\
\hline 98 & 3726. 37 & $5_{4}-5_{5}$ & 6. 62 & 26. 45 \\
\hline 80 & 3726.80 & $\mathrm{CO}_{2} \quad \mathrm{R}_{16}$ & & 26. 93 \\
\hline 60 & 3727.48 & $7_{0}-7_{1}$ & 0. 79 & 27. 67 \\
\hline 60 & 3728. 20 & $\begin{array}{ll}\mathrm{CO}_{2} & \mathrm{R}_{18}\end{array}$ & & 28. 25 \\
\hline 65 & 3729.54 & $\mathrm{CO}_{2} \quad \mathrm{R}_{20}$ & & 29. 56 \\
\hline 35 & 3729.93 & $\begin{array}{l}7_{1}-7_{-1} \\
4_{1}-4_{-1}\end{array}$ & $\begin{aligned} .84 * * \\
11.0\end{aligned}$ & $\begin{array}{l}\text { 29. } 99 \\
\text { 30. } 33\end{array}$ \\
\hline 70 & 3730.87 & $\begin{array}{ll}\mathrm{CO}_{2} & \mathrm{R}_{22}\end{array}$ & & 30. 83 \\
\hline 100 & 3732.00 & $\begin{array}{c}0-1_{-1} \\
\mathrm{CO}^{-}\end{array}$ & 26. 4 & $\begin{array}{l}32.00 \\
32.09\end{array}$ \\
\hline & & $6_{1}-6_{2}$ & 0.81 & 32. 36 \\
\hline 80 & 3 & $4_{-4}-3-2$ & 13. $2^{*}$ & 32.75 \\
\hline 70 & 3733. 37 & $\begin{array}{ll}\mathrm{CO}_{2} & \mathrm{R}_{26}\end{array}$ & & 33. 33 \\
\hline & & $\mathrm{CO}_{2} \quad \mathrm{R}_{28}$ & & $\begin{array}{l}34.53 \\
34.59\end{array}$ \\
\hline 100 & 3734.55 & $\begin{array}{l}6_{2}-6_{1} \\
5_{2}-5_{3}\end{array}$ & $\begin{array}{l}\text { 2. } 43 \\
6.46\end{array}$ & $\begin{array}{l}34.59 \\
35.05\end{array}$ \\
\hline & & $5_{3}-5_{2}$ & 2. 15 & 35. 45 \\
\hline 100 & 3735. 32 & $7_{1}-7_{0}$ & 0. 27 & 35. 45 \\
\hline & & $\begin{array}{rr}5_{0}- & 5_{1} \\
\mathrm{CO}_{2} & \mathrm{R}_{30}\end{array}$ & & $\begin{array}{l}35.50 \\
35.72\end{array}$ \\
\hline 100 & 3736. 67 & $4_{4}-4_{3}$ & 20.5 & $\begin{array}{l}36.71 \\
36.88\end{array}$ \\
\hline & & $\begin{array}{ll}\mathrm{CO}_{2} & \mathrm{R}_{32} \\
\mathrm{CO}_{2} & \mathrm{R}_{34}\end{array}$ & & $\begin{array}{l}36.88 \\
38.01\end{array}$ \\
\hline 95 & 3738. 31 & $2_{-1}-2_{0}$ & 5. 16 & 38. 19 \\
\hline & & $5-5$ & 2. $95^{*}$ & 38. 25 \\
\hline
\end{tabular}


TABLE 1. Absorption lines in the region of 2.7 $\mu$ due to $\mathrm{H}_{2} \mathrm{O}$ and $\mathrm{CO}_{2}-$ Continued

\begin{tabular}{|c|c|c|c|c|}
\hline \multicolumn{2}{|c|}{ Observed } & Identification & \multicolumn{2}{|c|}{ Calculated } \\
\hline $\begin{array}{l}\text { Inten- } \\
\text { sity }\end{array}$ & $\nu_{\mathrm{Vac}}$ & $J^{\prime} \tau-J^{\prime \prime} \tau$ & $\begin{array}{l}\text { Inten- } \\
\text { sity }\end{array}$ & $\nu$ \\
\hline & $\mathrm{cm}^{-1}$ & & & \\
\hline 45 & 3739. 17 & $\begin{array}{r}\mathrm{CO}_{2}-\mathrm{R}_{36} \\
8_{0}-8_{-1}\end{array}$ & 0. 24 & $\begin{array}{l}\text { 39. } 11 \\
\text { 39. } 19\end{array}$ \\
\hline 50 & 3740.34 & $\begin{array}{r}\mathrm{CO}_{2}-\mathrm{R}_{38} \\
6_{2}-6_{0}\end{array}$ & $83 * *$ & $\begin{array}{l}\text { 40. } 19 \\
40.60\end{array}$ \\
\hline 70 & 3740.84 & $\begin{array}{r}4_{0}-5_{-5} \\
4_{-3}-3_{-3}\end{array}$ & $\begin{array}{r}\text { 0. } 14 \\
\text { 43. } 1^{*}\end{array}$ & $\begin{array}{l}40.80 \\
40.83\end{array}$ \\
\hline 80 & 3741. 26 & $\begin{array}{c}4_{1}-4_{2} \\
\mathrm{CO}_{2} \mathrm{R}_{40}\end{array}$ & 5. 01 & $\begin{array}{l}\text { 41. } 33 \\
\text { 41. } 25\end{array}$ \\
\hline 25 & 3742.37 & $\begin{array}{ll}\mathrm{CO}_{2} & \mathrm{R}_{42}\end{array}$ & & 42. 28 \\
\hline 15 & 3742.87 & $\begin{array}{c}6_{-4}-6_{-6} \\
5_{1}-5_{0}\end{array}$ & $\begin{array}{l}\text { 1. } 65^{*} \\
\text { 2. } 03\end{array}$ & $\begin{array}{l}\text { 42. } 99 \\
\text { 44. } 10\end{array}$ \\
\hline 100 & 3743. 9 & $\begin{array}{l}4_{2}-4_{1} \\
3_{0}-3_{1}\end{array}$ & $\begin{array}{l}\text { 15. } 0 \\
21.5\end{array}$ & $\begin{array}{l}\text { 44. } 13 \\
\text { 44. } 44\end{array}$ \\
\hline 100 & 3745.1 & $\begin{array}{l}3_{2}-3_{3} \\
3_{3}-3_{2}\end{array}$ & $\begin{array}{l}\text { 33. } 6 \\
11.2\end{array}$ & $\begin{array}{l}\text { 44. } 62 \\
\text { 45. } 02\end{array}$ \\
\hline 60 & 3746. 23 & $\begin{array}{ll}2_{1} & 1_{1}\end{array}$ & 35. $8 *$ & 46. 21 \\
\hline 85 & 3747.37 & $5_{3}-5_{1}$ & 4. $00 * *$ & 47. 67 \\
\hline 100 & 3749. 18 & $\begin{array}{c}5_{-3}-4_{-1} \\
1_{0}-1_{1}\end{array}$ & $\begin{array}{l}9.21^{*} \\
35.8\end{array}$ & $\begin{array}{l}\text { 49. } 13 \\
49.15\end{array}$ \\
\hline 100 & 3749. 49 & $2_{1}-2_{2}$ & 15. 8 & 49. 49 \\
\hline 70 & 3750.84 & $4_{4}-4_{2}$ & 1. $38 * *$ & 50.83 \\
\hline 90 & 3751.31 & $\begin{array}{l}5_{-5}-4_{-3} \\
6-3-6\end{array}$ & 45. $0 *$ & 51. 40 \\
\hline 100 & 3752.14 & $\begin{aligned} 6_{-3}- & 6_{-5} \\
2_{2}- & 2_{1} \\
4_{3}- & 4_{1}\end{aligned}$ & $\begin{aligned} \text { 4. } 80 * \\
\text { 47. } 4 \\
\text { 4. } 15 * *\end{aligned}$ & $\begin{array}{l}52.06 \\
52.23 \\
52.67\end{array}$ \\
\hline 65 & 3753. 75 & $\begin{array}{l}2_{2}-1_{0} \\
5_{2}-5_{0}\end{array}$ & $\begin{array}{l}\text { 10. } 2^{*} \\
\text { 1. } 35^{* *}\end{array}$ & $\begin{array}{l}\text { 52. } 71 \\
\text { 53. } 75\end{array}$ \\
\hline 3 & 3754.47 & $3_{-3}-2_{2}$ & .066 & 54. 65 \\
\hline 12 & 3755.33 & $\begin{aligned} 3_{3}- & 4_{-2} \\
5_{-4}- & 4_{-4}\end{aligned}$ & 12. $0^{*} * 11$ & $\begin{array}{l}\text { 54. } 79 \\
\text { 55. } 31\end{array}$ \\
\hline 95 & 3756.55 & $3_{1}-3$ & 7. 20 & 56.55 \\
\hline $\begin{array}{l}10 \\
90\end{array}$ & $\begin{array}{l}3758.02 \\
3758.98\end{array}$ & $6_{0}-6_{-1}$ & 2. 19 & 59. 03 \\
\hline 99 & 3759.76 & $1_{1}-1_{n}$ & 12. 3 & 59. 62 \\
\hline 30 & 3762.34 & $\begin{array}{l}7_{-5}-7_{-7} \\
4_{-4}-3_{1}\end{array}$ & 2. $32 *$ & 60. 14 \\
\hline 20 & 3763. 61 & $\begin{array}{r}4-4-3_{1} \\
3_{0}-2_{0}\end{array}$ & $\begin{array}{l}.26 \\
9.98 *\end{array}$ & $\begin{array}{l}62.38 \\
63.65\end{array}$ \\
\hline 20 & 3764.54 & $7_{-7}-66_{-\cdot 2}$ & .018 & 64. 46 \\
\hline 98 & 3765.62 & $\begin{array}{l}7_{3}-7_{1} \\
4_{0}-4_{-1}\end{array}$ & $\begin{array}{l}61 \text { ** } \\
.28\end{array}$ & $\begin{array}{l}\text { 64. } 64 \\
65.78\end{array}$ \\
\hline 10 & 3766.58 & & 9.28 & 00.78 \\
\hline 3 & 3767.95 & $7_{2}-7_{n}$ & $20 * *$ & 68. 09 \\
\hline 20 & 3768. 47 & $6_{-6}-5_{-4}$ & 8. $64^{*}$ & 6356 \\
\hline 100 & 3769.72 & $\begin{array}{l}2_{0}-2_{-1} \\
6_{3}-6_{1}\end{array}$ & $\begin{aligned} \text { 16. } 3 \\
\text { 1. } 06^{* *}\end{aligned}$ & $\begin{array}{l}69.65 \\
69.7\end{array}$ \\
\hline 60 & 3770.17 & $7_{-1}-7_{-2}$ & 23 & 70. 05 \\
\hline 30 & 3771. 39 & $\begin{aligned} 0_{-5}- & 5_{-5} \\
5_{5}- & 5_{3}\end{aligned}$ & $\begin{array}{r}25.9 * \\
1.24 *\end{array}$ & $\begin{array}{l}\text { 7. } 58 \\
71.39\end{array}$ \\
\hline 5 & 3773. 72 & $6_{-4}-5_{-2}$ & 2. $85^{*}$ & 73.58 \\
\hline 35 & 3777.65 & $4_{-1}-3_{-1}$ & 23. $9 *$ & 77.85 \\
\hline 99 & 3779.41 & $\begin{array}{l}1-1-0 \\
5_{-1}-5\end{array}$ & 10. 0 & $\begin{array}{l}79.36 \\
79.68\end{array}$ \\
\hline 22 & 3781. 73 & $6_{-4}^{-1}-5_{1}$ & .089 & 81. 93 \\
\hline 90 & 3784.35 & $3_{-1}-3_{-2}$ & 2. 76 & 84. 49 \\
\hline 55 & 3784.97 & $7_{-7}-6_{-5}$ & 19. $1 *$ & 85. 01 \\
\hline 20 & 3786.02 & $\begin{array}{r}3_{1}-2_{-1} \\
8_{-2}-8_{-3}\end{array}$ & $\begin{array}{r}18.0^{*} \\
.20\end{array}$ & $\begin{array}{l}85.09 \\
86.23\end{array}$ \\
\hline 15 & 3786. 72 & & & \\
\hline 12 & 3789.08 & & & \\
\hline 10 & 3789. 43 & $5_{-2}-4_{-2}$ & 5. $50 *$ & 89. 36 \\
\hline 3 & 3792. 37 & $5_{2}-6_{-3}$ & .007 & 92.47 \\
\hline 5 & 3793. 63 & $7_{-5}-6_{-3}$ & 5. $80^{*}$ & 93.50 \\
\hline 99 & 3796. 13 & $2-1-1_{\text {ค }}$ & 12. 3 & 96.23 \\
\hline 80 & 3797. 50 & $6_{-2}-6_{-3}$ & 1. 45 & 97. 82 \\
\hline 30 & 3798. 78 & $3_{2}-2_{2}$ & 11. $2^{*}$ & 98.83 \\
\hline 30 & 3799. 66 & $66_{-3}-5_{-3}$ & 10. $9 *$ & 99.85 \\
\hline 90 & 3800.26 & $3_{3}-2_{1}$ & 33. $4 * *$ & 00. 26 \\
\hline
\end{tabular}

TABLE 1. Absorption lines in the region of $2.7 \mu$ due to $\mathrm{H}_{2} \mathrm{O}$ and $\mathrm{CO}_{2}-$ Continued

\begin{tabular}{|c|c|c|c|c|}
\hline \multicolumn{2}{|c|}{ Observed } & Identification & \multicolumn{2}{|c|}{ Calculated } \\
\hline $\begin{array}{l}\text { Inten- } \\
\text { sity }\end{array}$ & $\nu_{\mathrm{vac}}$ & $J^{\prime} \tau-J^{\prime \prime} \tau$ & $\begin{array}{l}\text { Inten- } \\
\text { sity }\end{array}$ & $\nu$ \\
\hline & $\mathrm{cm}^{-1}$ & & & \\
\hline 100 & 3801. 15 & $2_{-2}-1_{-1}$ & 52.0 & 01.15 \\
\hline 95 & 3802. 70 & $4_{-2}-4_{-3}$ & 4. 33 & 02.86 \\
\hline 100 & 3806. 74 & $22_{0}-1_{1}$ & 34.9 & 06. 76 \\
\hline 100 & 3815.96 & $\begin{array}{r}2_{1}-2_{-2} \\
3_{-2}-2_{-}\end{array}$ & $\begin{aligned} 0.34 \\
51.9\end{aligned}$ & $\begin{array}{l}15.58 \\
15.95\end{array}$ \\
\hline 60 & 3818.55 & $7_{-3}^{-2}-7_{-4}^{-1}$ & 0.18 & 18. 47 \\
\hline 92 & 3819.88 & $\begin{array}{l}4_{1}-5_{1} \\
3_{0}-3_{-3}\end{array}$ & $\begin{array}{r}22.1 \\
2.10\end{array}$ & $\begin{array}{l}18.52 \\
19.83\end{array}$ \\
\hline 100 & 3820.65 & $3_{-3}-2_{-2}$ & 19. 9 & 20. 74 \\
\hline 100 & 3821.70 & $3_{0}-?_{1}$ & 24. 2 & 21. 69 \\
\hline 55 & 3823. 09 & $5_{-3}-5_{-4}$ & 0.75 & 23. 39 \\
\hline 50 & 3824.23 & $4_{0}-3_{-2}$ & 2. $81 * *$ & 24. 20 \\
\hline 45 & 3825.50 & $4_{2}-3_{0}$ & 7. $13 * *$ & 25. 47 \\
\hline 95 & 3826.66 & $3_{1}-2_{2}$ & 8. 04 & 26. 68 \\
\hline 50 & 3827.94 & $4_{-1}-4_{-4}$ & 0.77 & 27. 90 \\
\hline 10 & 3828.61 & $5_{1}-5_{-5}$ & $33 * *$ & 28. 64 \\
\hline 99 & 3831.68 & $3_{-1}-2_{0}$ & 15. 9 & 31.58 \\
\hline 100 & 3835. 02 & $4_{-3}-3_{-2}$ & 17. 3 & 35.00 \\
\hline 100 & 3837. 99 & $4_{-4}-3_{-3}$ & 55.0 & 37. 77 \\
\hline & & $8_{-4}-8_{-5}$ & 0. 19 & 39. 47 \\
\hline 98 & 3840.11 & $4_{1}-3_{2}$ & 3. 79 & 39. 94 \\
\hline 98 & 3841.22 & $\begin{aligned} b_{-2}- & b_{-5} \\
4_{2}- & 3_{3}\end{aligned}$ & 11. 35 & $\begin{array}{l}40.09 \\
41.22\end{array}$ \\
\hline & & $6_{-4}-6_{-5}$ & 1. 15 & 43. 48 \\
\hline 99 & 3843.89 & $4_{-1}-3_{0}$ & 9. 85 & 43. 66 \\
\hline & & $6_{-1}-6_{-4}$ & 0. 16 & 44. 31 \\
\hline $\begin{array}{l}35 \\
15\end{array}$ & $\begin{array}{l}3845.06 \\
3846.60\end{array}$ & $\begin{array}{r}5_{0}-5_{-3} \\
6_{-1}-5_{-1}\end{array}$ & $\begin{array}{l}.62 \\
6.64 *\end{array}$ & $\begin{array}{l}44.90 \\
46.68\end{array}$ \\
\hline 30 & 3849. 35 & $7_{-2}-7_{-5}$ & 0. 31 & 48. 98 \\
\hline & & $\begin{aligned} 4_{4}-3_{2} \\
6_{-3}-6_{-6}\end{aligned}$ & $\begin{array}{l}\text { 7. } 3 * * \\
0.35\end{array}$ & $\begin{array}{l}49.44 \\
49.68\end{array}$ \\
\hline 80 & 3849.86 & $4_{1}-4_{-2}$ & 14 & 49. 71 \\
\hline 20 & 3850.54 & $4_{3}-3_{3}$ & 21. $9 * *$ & 49.76 \\
\hline 100 & 3852. 29 & $5_{-4}-4_{-3}$ & 42. 4 & 52.03 \\
\hline & & $4_{0}-3_{1}$ & 29.0 & 54. 00 \\
\hline 100 & 3854.3 & $5_{-5}-4_{-4}$ & 14. 4 & 54. 02 \\
\hline 60 & 3856.95 & $\begin{array}{r}4_{-2}-3_{-1} \\
3_{2}-3_{-1}\end{array}$ & $\begin{array}{r}43.6 \\
0.19\end{array}$ & $\begin{array}{l}54.03 \\
56.61\end{array}$ \\
\hline 95 & $3857 \quad 43$ & $5_{2}-4_{3}^{-1}$ & 3. 91 & 57. 22 \\
\hline & & $5_{3}-4_{4}$ & 1. 31 & 57. 40 \\
\hline 10 & 3858.32 & $8_{-3}-8_{-6}$ & 0.051 & 58. 11 \\
\hline 5 & 3859.24 & $6_{2}-6_{-4}$ & $.004 * *$ & 59.27 \\
\hline 98 & 3861. 87 & $5_{0}-4_{1}$ & 12. 26 & 61.86 \\
\hline 50 & 3862.52 & $7_{-5}-7_{-6}$ & 0. 21 & 62.52 \\
\hline 60 & 3863. 48 & $2_{2}-1_{-1}$ & .77 & 63.35 \\
\hline 90 & 3864.48 & $5_{1}-4_{2}$ & 4. 06 & 64. 24 \\
\hline 100 & 3865. 22 & $5_{-2}-4_{-1}$ & 24.8 & 65.07 \\
\hline 50 & 3866. 21 & $7_{-4}-7_{-7}$ & 0.53 & 66. 18 \\
\hline 100 & 3869. 37 & $6-5-5_{-4}$ & 9. 92 & 69. 37 \\
\hline 100 & 3870. 21 & $9_{-4}-9_{-7}$ & $\begin{array}{r}0.062 \\
30\end{array}$ & $\begin{array}{l}69.80 \\
70.13\end{array}$ \\
\hline 65 & 3871.65 & $5_{-1}^{-6}-4_{-3}$ & 3. $40 *$ & 70.83 \\
\hline 5 & 3872.64 & $6_{4}-5_{5}$ & 1. 35 & 71. 62 \\
\hline 98 & 3874.03 & $\begin{array}{l}5_{2}-4_{2} \\
5_{2}-4\end{array}$ & $\begin{array}{r}\text { 4. } 14 * * \\
12.4 * *\end{array}$ & $\begin{array}{l}73.89 \\
73.97\end{array}$ \\
\hline 100 & 3874. 53 & $5_{-3}-4_{-2}$ & 10.55 & 74.50 \\
\hline 5 & 3876. 66 & $7_{0}-7_{-3}$ & 0.065 & 76. 49 \\
\hline 3 & 3877. 52 & $10_{-6}-10_{-7}$ & 0.022 & 77.58 \\
\hline 100 & 3880.07 & $6_{1}-5_{2}$ & 1. 25 & 79. 94 \\
\hline 100 & 3880. 33 & $\begin{array}{l}5_{-1}-4_{0} \\
6_{-3}-5_{-2}\end{array}$ & 5. 60 & $\begin{array}{l}80.07 \\
80.27\end{array}$ \\
\hline 95 & 3881.05 & $6_{2}-5_{3}$ & 3. 74 & 81.01 \\
\hline 20 & 3881. 82 & $8_{-5}-8_{-8}$ & 0.077 & 81. 72 \\
\hline 80 & 3883. 27 & $6_{-1}-5_{0}$ & 2. 98 & 83. 18 \\
\hline 30 & 3884. 08 & $7_{4}-6_{5}$ & 0.32 & 83. 88 \\
\hline
\end{tabular}


TABLE 1. Absorption lines in the region of $2.7 \mu$ due to $\mathrm{H}_{2} \mathrm{O}$ and $\mathrm{CO}_{2}-$ Continued

\begin{tabular}{|c|c|c|c|c|}
\hline \multicolumn{2}{|c|}{ Observed } & Identification & \multicolumn{2}{|c|}{ Calculated } \\
\hline $\begin{array}{l}\text { Inten- } \\
\text { sity }\end{array}$ & $\nu_{\mathrm{vac}}$ & $J^{\prime} \tau-J^{\prime \prime} \tau$ & $\begin{array}{l}\text { Inten- } \\
\text { sity }\end{array}$ & $\nu$ \\
\hline & $\mathrm{cm}^{-1}$ & & & \\
\hline 100 & 3885.77 & $\begin{array}{c}7_{-6}-6_{-5} \\
6_{1}-5\end{array}$ & $\begin{array}{r}\text { 18. } 4 \\
6.10 * *\end{array}$ & $\begin{array}{l}85.68 \\
85.69\end{array}$ \\
\hline 100 & $\begin{array}{l}3886.08 \\
3886.02\end{array}$ & $7_{-7}-6_{-6}$ & 6. 10 & 86.08 \\
\hline & 388730 & $6_{1}-6_{-2}$ & 0. 026 & 37. 39 \\
\hline 10 & 3887.30 & $3_{3}-3_{-2}$ & .033 & 88.00 \\
\hline 100 & 3891. 30 & $6-4-5_{-3}$ & 19. 7 & 91. 27 \\
\hline 85 & 3891.92 & $6_{0}-5_{-2}$ & 1. $49 * *$ & 91.33 \\
\hline 50 & 3892.80 & $\begin{array}{l}3_{1}-2_{-2} \\
5-4\end{array}$ & $\begin{array}{l}0.54 \\
3.15^{*}\end{array}$ & $\begin{array}{l}92.77 \\
93.57\end{array}$ \\
\hline 70 & 3894. 13 & $7_{2}-6_{3}$ & .84 & 94.08 \\
\hline & & $4_{1}-3_{-3}$ & $0.60 * *$ & 94. 19 \\
\hline 8 & 3895. 18 & $8_{6}-7_{7}$ & .042 & 94.97 \\
\hline 5 & 3896.88 & $9_{-7}-9_{-8}$ & .031 & 97.01 \\
\hline 10 & 3897.62 & $9_{-6}-9_{-9}$ & 0.093 & 97.85 \\
\hline 65 & 3898.16 & $6_{2}-5_{0}$ & $.13 * *$ & 98. 14 \\
\hline 100 & 3899.38 & $\begin{array}{l}5_{2}-5_{-1} \\
6_{0}-5_{1}\end{array}$ & 8.75 & 99.21 \\
\hline & & $7_{-4}-6-3$ & 9. 62 & 99.54 \\
\hline & & $7_{0}-6_{0}$ & 2. $40^{*}$ & 01. 41 \\
\hline 100 & 3901.91 & $8_{-7}-7_{-6}$ & $\begin{array}{l}\text { 3. } 30 \\
0.90\end{array}$ & 01. 78 \\
\hline 98 & 3902.15 & $7_{0}^{8}-6_{1}^{-7}$ & 2. 41 & 02.14 \\
\hline 100 & 3904.22 & $7_{-2}-6_{-1}$ & 5. 18 & 04. 21 \\
\hline (0) & 2005517 & $6_{-2}-5_{-1}$ & 14.55 & 04. 24 \\
\hline 60 & $3905.51\}$ & $7_{1}-6_{2}$ & 0.80 & 05.51 \\
\hline 90 & 3906. 15 & $\begin{array}{l}8_{4}-6_{-4}^{5} \\
7_{-5}-6_{-4}\end{array}$ & 3. 47 & $\begin{array}{l}06.01 \\
06.08\end{array}$ \\
\hline 3 & 3910.32 & $4_{3}-4_{-3}$ & $0.10 * *$ & 10. 33 \\
\hline 5 & 5910. 32 & $9_{0}-9_{-3}$ & .004 & 10. 47 \\
\hline 5 & 3912. 86 & $10_{-8}-10_{-9}$ & .030 & 12. 80 \\
\hline 8 & 3914. 06 & $8_{-1}-7_{-1}$ & . $99^{*}$ & 14. 09 \\
\hline 80 & $3916 \quad 35$ & $\begin{array}{c}6_{3}-5_{3} \\
8_{-5}-7_{-4}\end{array}$ & 4. $78^{*}$ & $\begin{array}{l}16.1 \\
16.35\end{array}$ \\
\hline 00 & & $8_{1}^{-5}-7_{2}^{-4}$ & 0. 16 & 16. 42 \\
\hline 92 & 3917.32 & $8_{2}-7_{3}$ & .48 & 17. 17 \\
\hline & & $9_{-8}-8_{-7}$ & 6. 36 & 17. 42 \\
\hline 90 & 3920. 12 & $8_{-6}-7_{-5}$ & 4. 99 & 20. 13 \\
\hline 60 & 3923. 46 & $7_{1}-6_{-1}$ & 2. $70^{* *}$ & 23. 38 \\
\hline 40 & 3923. 70 & $8_{-1}-7_{0}$ & 0.41 & 23. 70 \\
\hline 60 & 3924. 38 & $8_{-3}-7_{-2}$ & .84 & 24. 43 \\
\hline 88 & 3925. 20 & $7_{-1}-6_{0}$ & 1. 63 & 25. 16 \\
\hline 15 & 3928. 19 & $\begin{array}{c}y_{-3}-0_{-2} \\
9-8_{3}\end{array}$ & $\begin{array}{l}2.49 \\
0.101\end{array}$ & $\begin{array}{l}25.20 \\
28.11\end{array}$ \\
\hline 75 & $3929 \quad 53$ & $4_{0}-3_{-3}$ & 1. 53 & 29. 39 \\
\hline$\gamma$ & & $5_{3}-5_{-2}$ & 0.021 & 29.45 \\
\hline 65 & 3930.83 & $8_{0}-7_{1}$ & 1. 20 & 30.74 \\
\hline 88 & 3932.42 & $9_{-6}-8_{-5}$ & 2. 12 & 32.42 \\
\hline 85 & 3932. 76 & $10_{-10}-9_{-9}$ & 2. 74 & 32.67 \\
\hline 55 & 3934. 25 & $9_{-7}-8_{-6}$ & 0.71 & 34. 25 \\
\hline 10 & 3935. 29 & $3_{3}-2_{0}$ & .094 & 35. 10 \\
\hline 25 & 3938. 42 & $\begin{array}{l}9_{0}-8_{1} \\
6_{2}-6_{-3}\end{array}$ & $\begin{array}{l}.217 \\
.054\end{array}$ & $\begin{array}{l}38.30 \\
38.43\end{array}$ \\
\hline 15 & 3939.24 & $7_{3}-6_{1}$ & 2. $15^{* *}$ & 39. 11 \\
\hline & & $7_{-1}-6_{-3}$ & 1. $82^{*}$ & 39. 22 \\
\hline 6 & 3940.80 & $9_{1}-8_{2}$ & 0.072 & 40. 65 \\
\hline 90 & 3942. 86 & $8_{-4}-7_{-3}$ & 3. 26 & 42. 70 \\
\hline 45 & 3944. 49 & $9_{-2}^{-4}-8_{-1}^{-3}$ & .51 & 44. 40 \\
\hline 55 & 3947. 39 & $10_{-7}-9_{-6}$ & . 28 & 47. 39 \\
\hline 60 & 3947. 59 & $11_{-10}-10_{-9}$ & 1. 06 & 47.53 \\
\hline 55 & 3948. 37 & $10_{-8}-9_{-7}$ & .84 & 48.35 \\
\hline 80 & 3950.12 & $8_{-2}-7_{-1}$ & 2. 23 & 50.02 \\
\hline 25 & 3950.51 & $3_{2}-2_{-1}$ & .202 & 50.54 \\
\hline 10 & 3952. 57 & & & \\
\hline 40 & 3953. 28 & $4_{2}-\widehat{3}_{-1}$ & .57 & 53. 26 \\
\hline
\end{tabular}

TABLE 1. Absorption lines in the region of $2.7 \mu$ due to $\mathrm{H}_{2} \mathrm{O}$ and $\mathrm{CO}_{2}-$ Continued

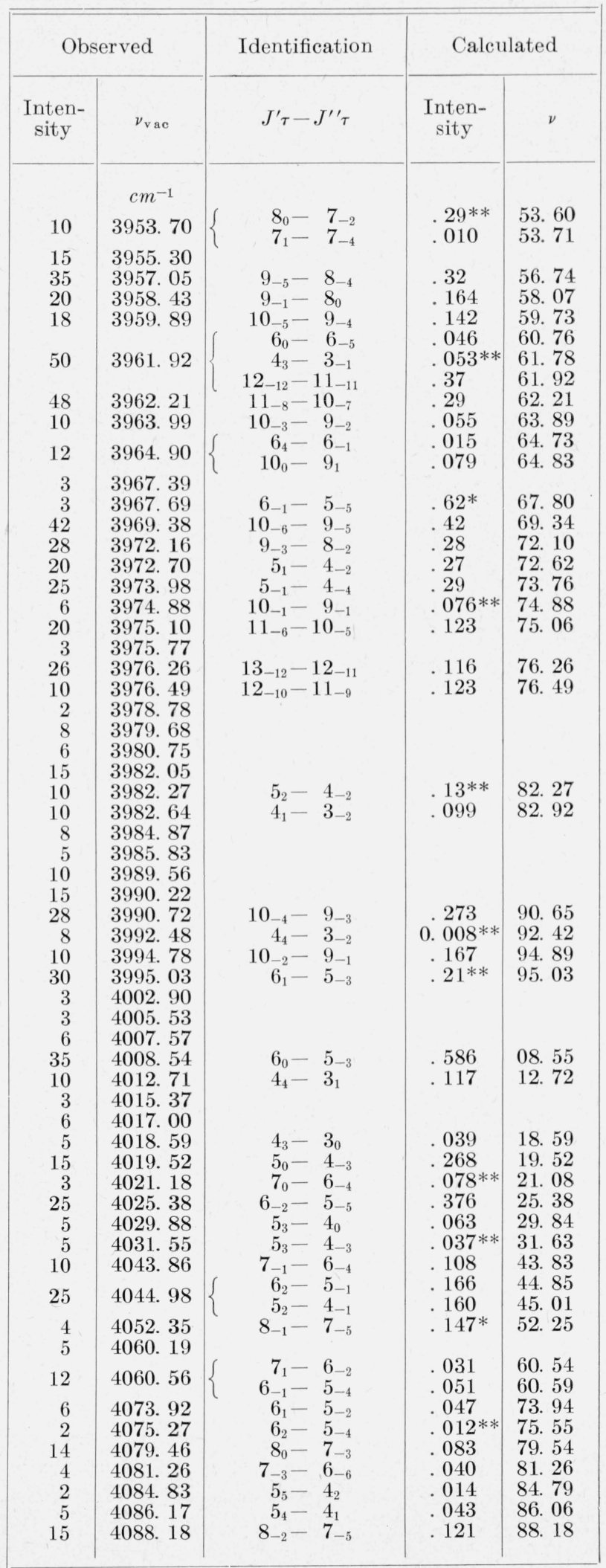


The third and fourth columns of table 1 give the identifications of the line, or, if several transitions overlap to give an unresolved blend, the strongest such components are listed. The entries in these columns take three forms. (a) First-order $\mathrm{H}_{2} \mathrm{O}$ lines. These may belong to either the $\nu_{3}$ or the $\nu_{1}$ fundamental. In either case we list first the rotational quantum numbers $J \tau^{\prime}$ belonging to the upper vibrational level, and next the corresponding rotational quantum numbers $J \tau^{\prime \prime}$ of the lower (vibrationless) level. $\nu_{3}$ lines may be distinguished from $\nu_{1}$ lines by the different selection rules that apply. These may most simply be stated as follows: in $\nu_{3}$ (a so-called type A band, where the change of dipole moment is along the least axis of inertia) the parity of $\tau$ must change (odd $\longleftrightarrow$ even); whereas in $\nu_{1}$ (a type B band, where the change of dipole moment is along the intermediate axis of inertia) the parity of $\tau$ is fixed (odd $\longleftrightarrow \rightarrow$ odd, or even $\longleftrightarrow$ even). (b) Second-order $\mathrm{H}_{2} \mathrm{O}$ lines. The entry here is the wave number reported by Nelson [7] for the line in question, followed by the symbol $/ 2$. (c) $\mathrm{CO}_{2}$ lines. The entry for these reads $\mathrm{CO}_{2} R_{J}$ (or $P_{J}$ ), where the $J$ signifies the rotational quantum number $J^{\prime \prime}$ of the ground state, and $R$ or $P$ is the conventional symbol for the $R$ branch $\left(J^{\prime}=J^{\prime \prime}+1\right)$ or the $P$ branch $\left(J^{\prime}=J^{\prime \prime}-1\right)$, respectively. As the two $\mathrm{CO}_{2}$ bands, whose centers fall near $3,613 \mathrm{~cm}^{-1}$ and $3,715 \mathrm{~cm}^{-1}$, do not overlap each other, no differentiation between them is given in the table.

The fifth and sixth columns of table 1 give calculated values of the intensity and frequency. The intensity values in the fifth column, when referring to first-order $\mathrm{H}_{2} \mathrm{O}$ lines are relative values within each band, $\nu_{3}$ or $\nu_{1}$. The latter lines are distinguished by the symbol $*$, and a few lines of $\nu_{1}$, for which abnormally high intensities are expected because of the perturbation phenomenon to be discussed in greater detail later, are further distinguished by a double asterisk $(* *)$. The calculated intensity has the following significance. It is proportional to the product of the "line strength", or probability of rotational transition $J_{\tau^{\prime}}-J_{\tau^{\prime \prime}}$, into the Boltzmann factor $g e^{-E \prime \prime / k T}$, where $g$ is the statistical weight (3 for odd $\tau, 1$ for even $\tau$ ), $E^{\prime \prime}$ is the rotational term. value of the ground level $J_{\tau^{\prime \prime}}$ (these are listed in table 2), $k$ is the Boltzmann constant, and $T$ is the Kelvin temperature. In making the calculation, the line strengths were taken from the very useful tabulation of Cross, Hainer, and King [8], for the case of an a symmetric rotor with the asymmetry parameter $\kappa=-0.5$ in both upper and lower states. Hence the results should not precisely apply to the $\mathrm{H}_{2} \mathrm{O}$ bands in question, where $\kappa^{\prime \prime}=-0.437$ and $\kappa^{\prime}=-0.400$ for $\nu_{3}$ and -0.425 for $\nu_{1}$, but should be a good approximation to the true line strengths. Furthermore, in the calculation the temperature was taken as $267^{\circ} \mathrm{K}$, for which the intensities had already been calculated [4] rather than the actual temperature of about $291^{\circ} \mathrm{K}$; this would introduce an appreciable error only in a few of the transitions of highest $E^{\prime \prime}$. The absolute absorption intensity of a line, in addition to the relative factors tabulated, would depend upon the number of molecules in the absorbing path in the ground vibrational state, which is a constant of the experiment; upon the line width and slit width, which is approximately a constant of the experiment; upon the frequency, which varies by little more than 10 percent from one limit of the band to the other; and upon a factor representing the effective change of dipole moment in the vibrational transition. This last factor should be a constant for all lines of $\nu_{3}$ and another constant for all lines of $\nu_{1}$, to the approximation that the vibrational-rotational wave functions may be separated into vibrational and rotational portions. From a comparison of the observed intensities with those calculated it will be noted that the vibrational transition probability for $\nu_{3}$ must be considerably stronger than that for $\nu_{1}$. The proportionality factor seems to vary, however, being greater for lines in the $R$ branches, where it is of the order of $50: 1$, than for lines in the $P$ branches, where it is of the order of 15:1. This point will be discussed at greater length.

For second-order $\mathrm{H}_{2} \mathrm{O}$ lines, the entry in column five of table 1 is the reported intensity, from reference [7], enclosed in parentheses. No entry is given for the intensity of the $\mathrm{CO}_{2}$ lines; it is sufficient to note that a regular increase in intensity with $J$ is expected, up to $J=20$, followed by a regular decrease. The weakest lines, $R_{0}, R_{40}, P_{40}$, etc., should have an intensity of the order of one-twentieth that of the strongest lines.

The calculated frequencies of the first-order $\mathrm{H}_{2} \mathrm{O}$ lines in column six of table 1 , are derived from the term values for the corresponding rotational states, which are listed in table 2. A calculated frequency is the difference between the two energy levels, $J \tau^{\prime}$ and $J_{\tau^{\prime \prime}}$, involved in the transition. The term values for the ground state are those derived from the analysis of a large number of rotation-vibration bands [4]; they differ only very slightly from previously published values [9]. The term values for the upper levels $\nu_{3}(001)$ and $\nu_{1}(100)$ are derived from the present data. As each upper level gives rise to from two to six lines that are observably strong, and of which usually three or four are free from overlapping, the upper term values are obtained by adding the known lower term value to the observed $\nu$ for each line, and averaging the reśults. The concordance of the observed and calculated $\nu$ 's, in which the disagreement rarely exceeds $0.2 \mathrm{~cm}^{-1}$, thus indicates the consistency of the measurements. The correctness of the analysis is more definitely indicated by the agreement in relative intensities, and by the degree to which the observed term values of table 2 agree with theoretical calculations, which will be discussed later.

The calculated frequencies of the second-order $\mathrm{H}_{2} \mathrm{O}$ lines are obtained in the same manner, the identification of the quantum transitions and the term values being given elsewhere $[7,4]$; the calculated frequencies were then divided by 2 . The calculated frequencies for the $\mathrm{CO}_{2}$ lines were obtained by fitting the data to the well-known formula for a linear molecule [10]. The value of $B^{\prime \prime}, 0.3904 \mathrm{~cm}^{-1}$, was taken from the analysis of other well-resolved 
TABLE 2. Energy levels $\left(\mathrm{cm}^{-1}\right)$ of $\mathrm{H}_{2} \mathrm{O}$ leading to lines in table 1

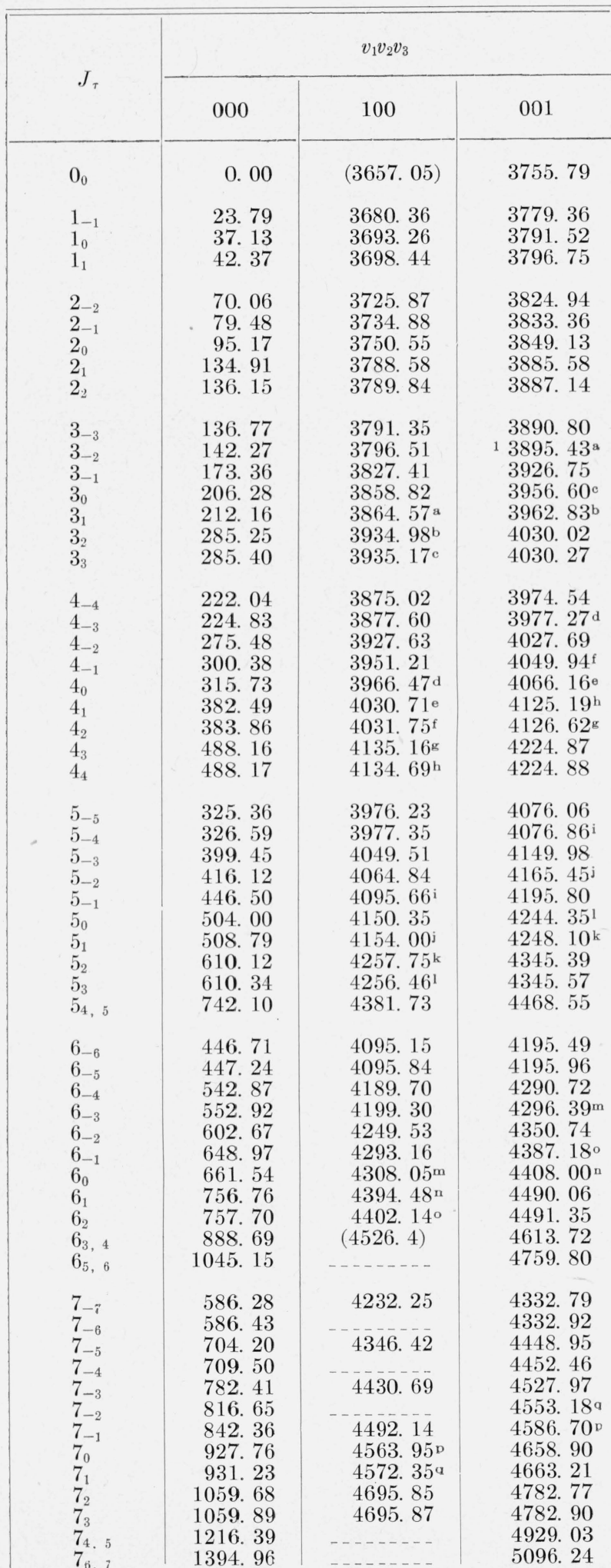

TABLE 2. Energy levels $\left(\mathrm{cm}^{-1}\right)$ of $\mathrm{H}_{2} \mathrm{O}$ leading to lines in table 1-Continued

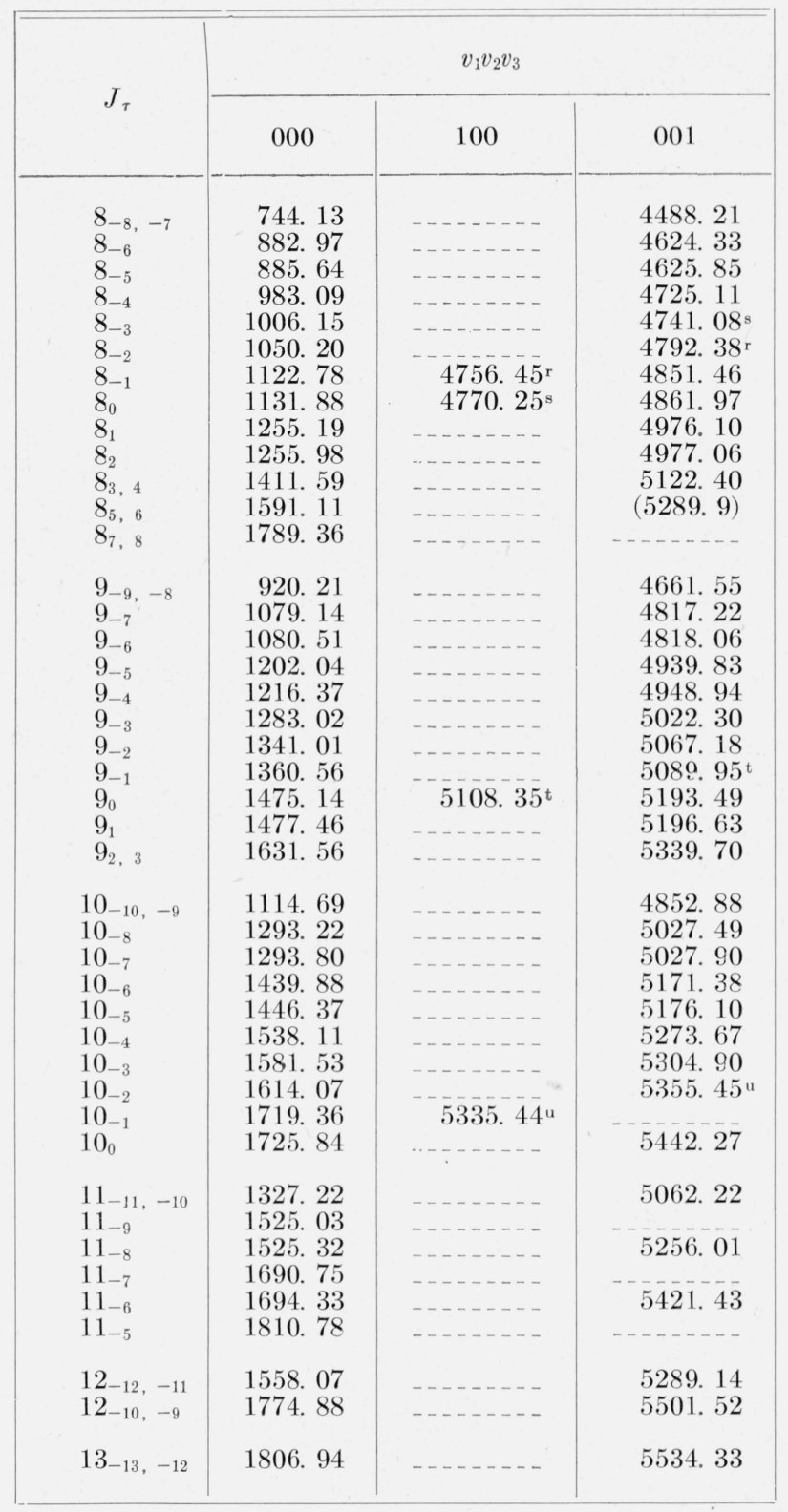

1 The letters a, b. etc., identify pairs of levels in (100) and (001) that are particularly strongly connected by perturbation interactions. See text.

bands of this molecule [11]; the resulting values of $B^{\prime \prime}-B^{\prime}$ and $\nu_{0}$ are listed in table 3 . The observed values of these parameters are in good agreement with the values calculated from an empirical equations whose constants fit the other $\mathrm{CO}_{2}$ bands. ${ }^{2}$

2 In a paper published after the completion of this work, Jones and Bell (Phys. Rev. 79, $1004(1950)$ ) have reported measurements of the $\mathrm{C}_{2}$ bands in a vacuum grating spectrometer. The constants they derive are in excellent agreement with ours for $B^{\prime}-B^{\prime \prime}$, but differ by about $1 \mathrm{~cm}^{-1}$ for the $\nu_{0}$ 's. Their values of $B^{\prime \prime}$ also do not agree well with the published values we have adopted [11]. These discrepancies must be due to different methods used in establishing the wavelength scales; it is difficult to believe that our values, based on accurately known lines in higher orders, could be in error by as much as $1 \mathrm{~cm}^{-1}$. 


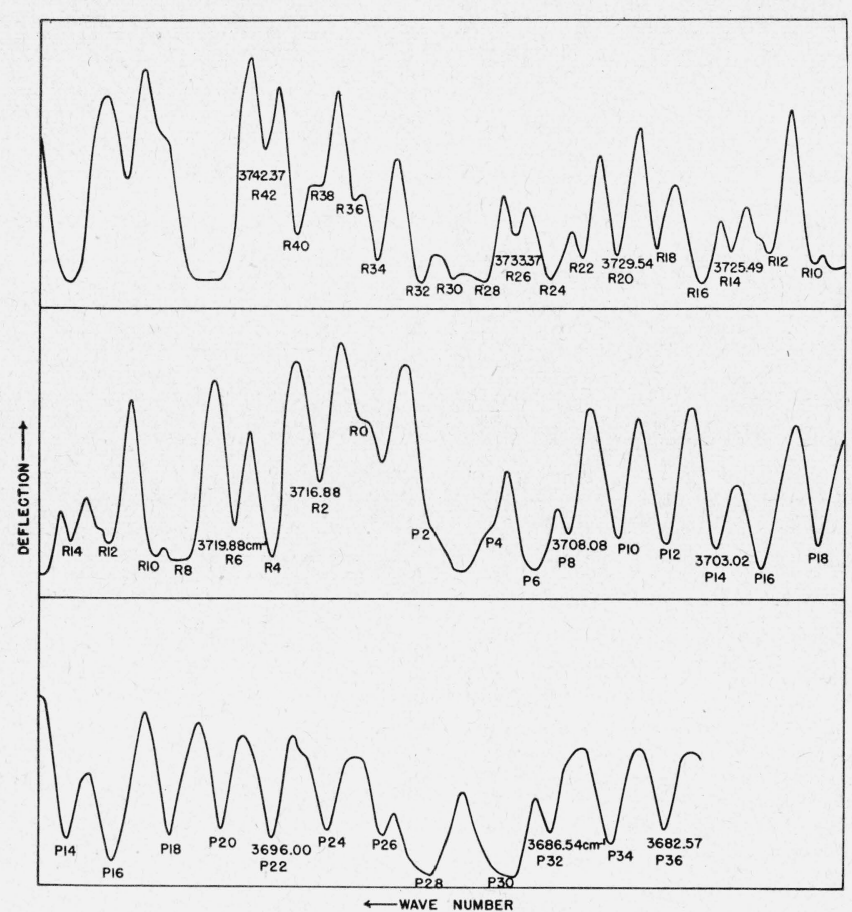

FIGURE 5. Observed absorption enhanced by absorption cell containing $\mathrm{CO}_{2}, 3,750$ to $3,680 \mathrm{~cm}^{-1}$.

TABLE 3. Constants of $\mathrm{CO}_{2}$ bands in 2.7- $\mu$ region

\begin{tabular}{|c|c|c|c|c|c|c|}
\hline \multirow{2}{*}{$v_{1}$} & \multirow{2}{*}{$v_{2}$} & \multirow{2}{*}{$v_{3}$} & \multicolumn{2}{|c|}{$\nu_{0}$} & \multirow{2}{*}{$\frac{B^{\prime \prime}}{\text { Observed }}$} & \multirow{2}{*}{$\frac{B^{\prime}}{\text { Calculated }}$} \\
\hline & & & Observed & Calculated & & \\
\hline$\left\{\begin{array}{l}0 \\
1\end{array}\right.$ & $\begin{array}{l}2 \\
0\end{array}$ & $\begin{array}{l}1 \\
1\end{array}$ & $\begin{array}{c}\mathrm{cm}^{-1} \\
3612.91 \\
3714.59\end{array}$ & $\begin{array}{c}c m^{-1} \\
3613.64 \\
3714.76\end{array}$ & $\begin{array}{c}c m^{-1} \\
0.0027_{9} \\
.0030_{9}\end{array}$ & $\begin{array}{c}c m^{-1} \\
0.00274 \\
.00312\end{array}$ \\
\hline
\end{tabular}

The absorption due to $\mathrm{CO}_{2}$, when an absorption cell filled with this gas was added to the optical path, is shown in figure 5, covering the region of the higherfrequency band of the resonance doublet, from 3,680 to $3,748 \mathrm{~cm}^{-1}$. Comparison with the corresponding region in figures 2 and 3 shows a marked enhancement of the lines that have been attributed to $\mathrm{CO}_{2}$. Figure 5 also shows the rotational numbering for the band and makes it clear that the center of the band has been correctly located.

\section{Discussion}

The rotational analysis of the $\nu_{3}$ band is rather complete, in that nearly all the theoretically expected lines of intensity 0.2 or greater (on the adopted relative scale on which the strongest line, $4_{-4}-3_{-3}$, has intensity 55.0) have been located. A few lines as weak as 0.03 appear, giving about 5-percent maximum absorption, in regions where there is no overlapping from stronger lines. The identified levels of $\nu_{3}$ include all levels with $J^{\prime}=8$, and a number of the lower-energy levels of higher $J$, as listed in table 2 . The $\nu_{1}$ analysis is less complete, because of the much lower intensity of the lines of this band, particularly in the $R$ branch, where our measurements are most satisfactory. Many of the strongest lines of the $P$ branch of $\nu_{1}$, and some of the higher- $J$ lines of the $P$ branches of $\nu_{3}$, fall in the region beyond 3,500 $\mathrm{cm}^{-1}$, to which our measurements do not extend. These lines may be recognized in other published and unpublished measurements of this region [12], overlapping the $R$ branches of the weaker overtone band $2 \nu_{2}$. The known values of $\nu_{1}$ used here are partially based on the longer wavelength data. Table 1 shows that nearly 98 percent of the lines, and all of the stronger ones, are satisfactorily accounted for.

As an illustration of the agreement between observation and the analysis, figure 6 represents a portion of the observed spectrum, together with the calculated spectrum. The latter is represented by lines at the calculated frequency, whose length is the theoretical relative intensity, on a scale that exaggerates the importance of the weaker lines. The region depicted, between 3,845 to $3,895 \mathrm{~cm}^{-1}$, is dominated by the stronger lines of the $R_{0}$ branch from $J^{\prime}=4$ to $J^{\prime}=7$, with calculated intensities $>3$. These give observed absorptions of 80 percent or greater; lines with intensity $>10$ show practically complete absorption; and the overlapping of three such lines, with calculated $\nu$ 's $3854.0 \pm 0.1 \mathrm{~cm}^{-1}$, gives rise to a region of complete absorption over $1 \mathrm{~cm}^{-1}$ broad. The weaker lines in the region include the lowest members of the $\nu_{3} R_{2, \overline{1}}$ branch (e. g., $2_{2}-1_{-1}$; Int calc $=0.77$, Int $o b s=60 \%$ ), a few lines of the $\nu_{3} \Omega_{2, \overline{1}}$ branch (e. g., $7_{0}-7_{-3}$; Int calc $=0.065$, Int $\mathrm{obs}=5 \%$ ); and some of the stronger members of the $R$ branches of $\nu_{1}$. Many of the latter give quite weak absorption; for example $6_{-1}-5_{-1}$, with Int calc $=6.6$, shows only 15-percent absorption. It is from comparisons of this kind that the statement was made above that the intensity of $\nu_{1}$ relative to $\nu_{3}$, in the $R$ branch, was of the order of $1: 50$.

There are, however, some isolated members of the $\nu_{1} R$ branches that show much more intense absorption. For example, the strong absorption $(85 \%)$ at $3891.92 \mathrm{~cm}^{-1}$ is assigned to $6_{0}-5_{-2}$ whose calculated intensity is only 1.99 , much less than the $6_{-1}-5_{-1}$ line. Similariy the pair $5_{3}-4_{1}, 5_{2}-4_{2}$ at $3874.0 \mathrm{~cm}^{-1}$ show an observed absorption more nearly characteristic of $\nu_{3}$ than of $\nu_{1}$. These observations find a natural interpretation in the perturbation of a rotationvibration level of $\nu_{1}$ by a close-lying one of $\nu_{3}$. It wil! be noted in table 2 that letters have been suffixed to certain levels in pairs, one in each vibration state. The pairs have the characteristic that they are of the same $J$, the same total (vibrational $X$ rotational) symmetry properties, and are of approximately the same energy $\left(<30 \mathrm{~cm}^{-1}\right.$ apart $)$. Such pairs are subject to a mutual perturbation, induced by the Coriolis forces interacting between the vibrations and the rotation [13]. The perturbation results in a displacement of the frequencies, and a mixing of the wave-functions of the two levels. The frequency shifts will be discussed quantitatively later; 


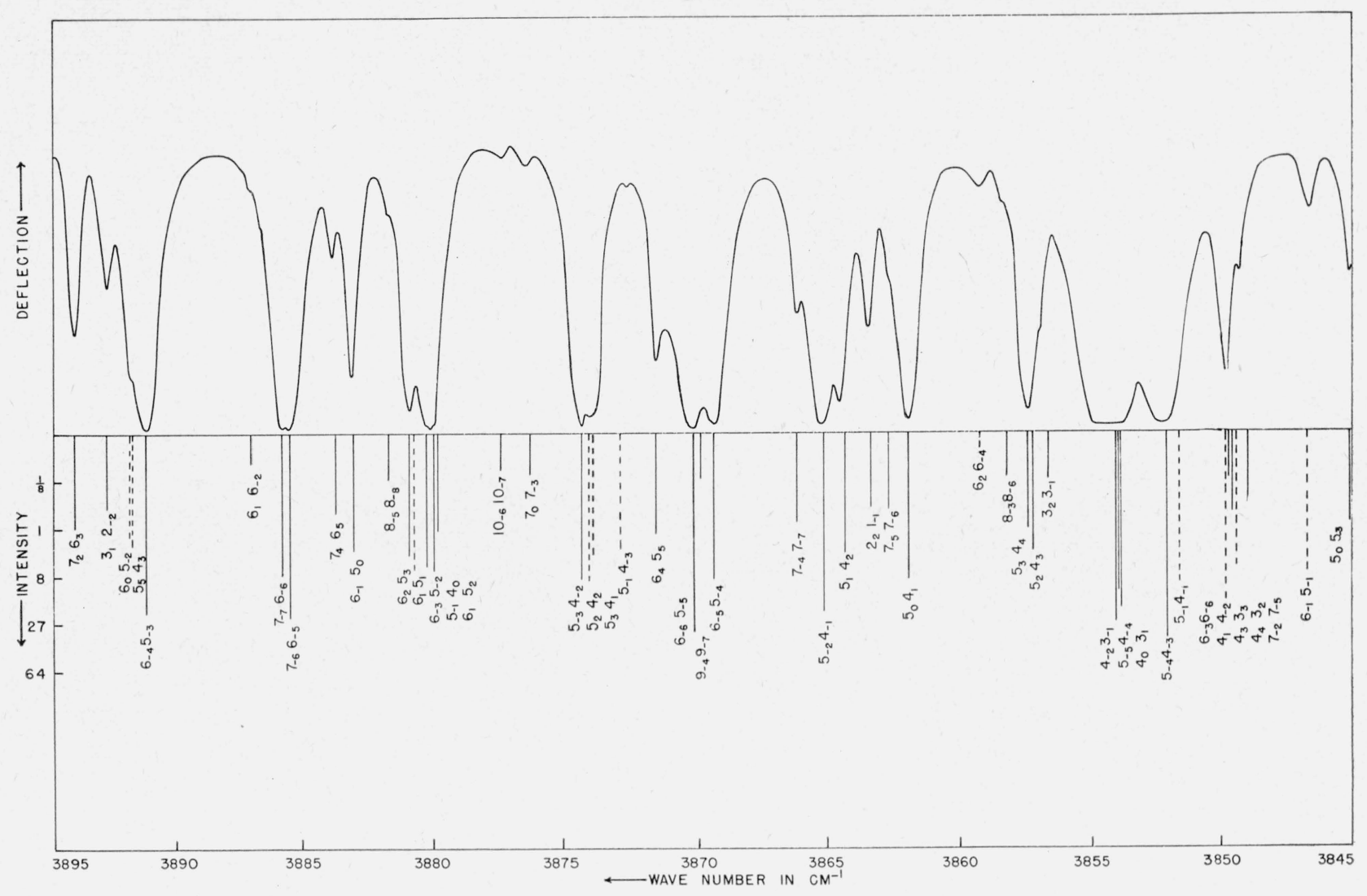

FIGURE 6. Comparison of observed and theoretically calculated spectra, 3,895 to $3,845 \mathrm{~cm}^{-1}$.

they are small, but are apparent in table 2 in such instances as levels $k$ and $l$ of (100), which are inverted from their normal order, or $n$ and $o$ of (100) which are separated by $7.66 \mathrm{~cm}^{-1}$ instead of $1 \mathrm{~cm}^{-1}$, which would be normal for the close doublet $6_{1}-6_{2}$. These are precisely the levels that give rise to the $\nu_{1}$ lines of abnormally high intensity, as indicated by $* *$ in table 1 . The perturbation has, for example, caused the $\nu_{1}$ line $6_{0}-5_{-2}$ to partially merge its identity with the $\nu_{3}$ line $6_{-3}-\tilde{0}_{-2}$, with a resultant merger of the intensity of the two lines. Even more striking is the appearance of such a weak transition as $\nu_{1} 6_{2}-66_{-4}$ (Int calc $=0.004)$; this is due to its perturbation by $\nu_{3} 6_{-1}-6_{-4}($ Int calc $=0.16)$.

Figure 7 is a similar illustration of the agreement between observation and calculation in the region 3,775 to $3,25 \mathrm{~cm}^{-1}$. The strong lines in this region are the first members of the $R_{0,1}$ branch of $\nu_{3}$. Some of the very weak lines of the $\nu_{3} R$ and $P$ branches appear. The relatively low intensity of the $\nu_{1} R$ branch lines is again to be noted.

Figure 8 similarly depicts the situation from 3,505 to $3,590 \mathrm{~cm}^{-1}$. Here the second-order radiation is a complicating factor in evaluating relative intensities, but it is clear that in this region, which includes higher- $J$ members of the $P$ branch of $\nu_{3}$ and the stronger members of the $P$ branch of $\nu_{1}$, the relative intensity of $\nu_{1}: \nu_{3}$ is considerably higher than in the $R$ branches. It seems certain that this anomaly occurs in $\nu_{1}$ rather than $\nu_{3}$; similar anomalous inten- sities have also been found in other of the type $B$ bands of $\mathrm{H}_{2} \mathrm{O}$ [4]. For example, the type $B$ band (130) has a $P$ branch that is about one-half the observed intensity of the $P$ branch of the adjoining type $A$ band (031), whereas the relative intensities in the corresponding $R$ branches are about 1:10. A very similar anomaly has also been observed in $\mathrm{H}_{2} \mathrm{~S}$ [14], where the $R$ branch of the $\nu_{3}$ fundamental is very much stronger than the $P$ branch. A quantitative explanation of this behavior cannot be offered but it undoubtedly is associated with the breakdown of the assumption of separability of the molecular wave-function into vibrational and rotational portions. The interaction terms in the molecular potential function, which result in different effective moments of inertia for different vibrational states, will also affect the transition probabilities, and it may be expected that anomalous intensities will result. This effect should, however, be roughly constant for all transitions of a similar type, so that the relative intensities within a particular branch should agree with those calculated on the assumption of separability, in agreement with observation.

Another way in which the correctness and completeness of the rotational analysis may be demonstrated is by arranging the lines of a given branch in series. This is done in table 4 for the $R_{0,1}$ branch of $\nu_{3}$. The observed $\nu$, observed intensity, and calculated intensity are listed for all lines, arranged vertically in order of increasing $J$, horizontally in 


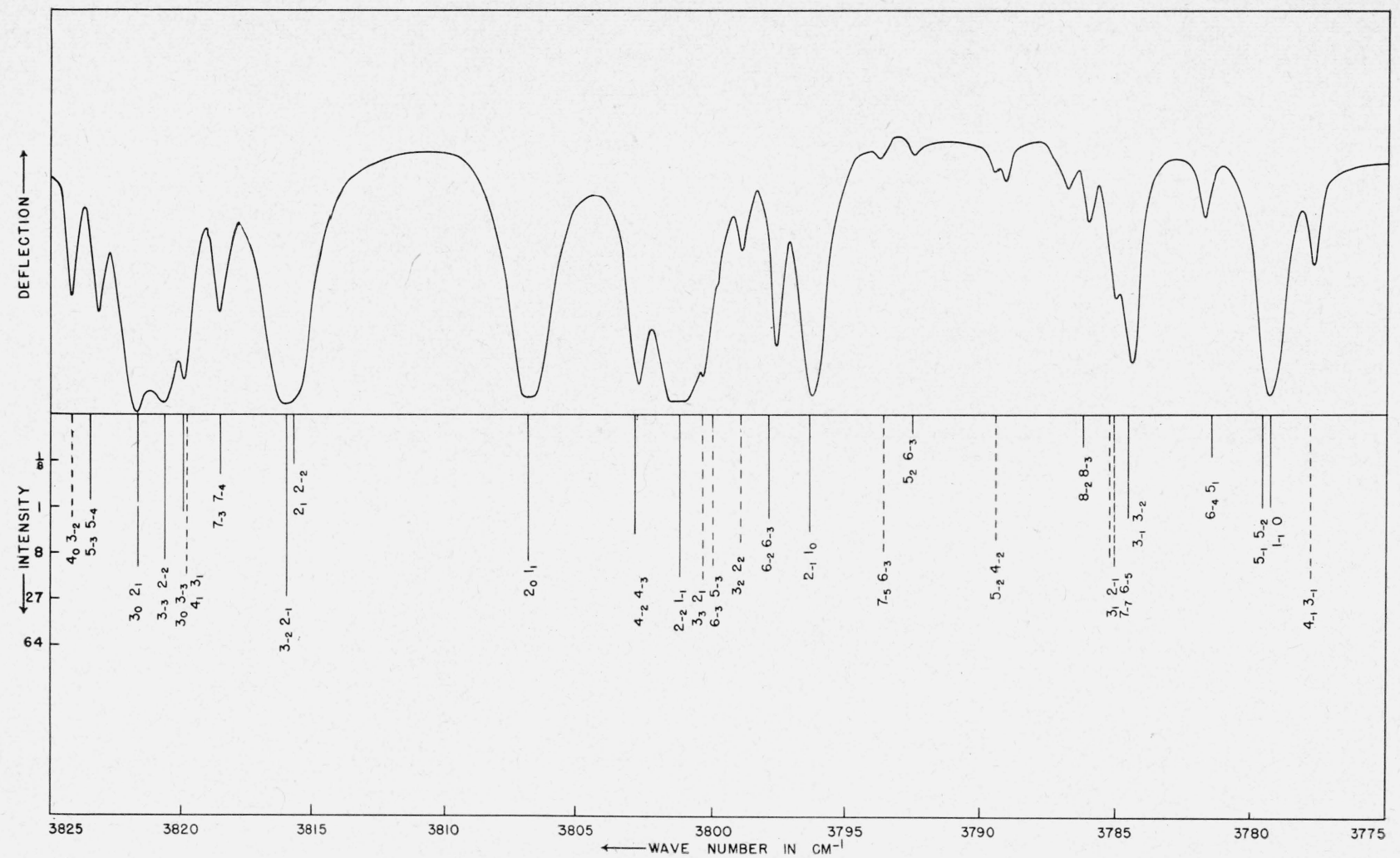

Figure 7. Comparison of observed and theoretically calculated spectra, 3,825 to $3,775 \mathrm{~cm}^{-1}$.

order of increasing $\tau$. Regularities in both position and intensity are apparent, except that for a few of the $\tau$ series there is an irregularity, which is seen to be associated with the perturbation of the $\nu_{3}$ levels by close-lying $\nu_{1}$ states. Similar series arrangements may be made of the lines of the other branches and of $\nu_{1}$, but will not be reproduced here.

In table 4, spaces have been left for lines that have not yet been identified. By following the indicated regularities, provisional assignments may readily be made for the stronger of these expected lines, and some of these have been listed in table 4 . In order to confirm the assignments, it would be necessary to locate the lines of corresponding $J_{\tau^{\prime}}$ in other branches. As these are either too weak to observe, or fall outside the present limits of observation, such lines and levels have not been included in tables 1 and 2. By making observations of the absorption of heated water vapor, over the spectral range 3,350 to $4,300 \mathrm{~cm}^{-1}$, it should be possible to confirm these and extend the analysis to higher energies; it is hoped to do this in the near future. Observations $[4,12]$ of the absorption of the whole atmosphere (between 3,300 to $3,500 \mathrm{~cm}^{-1}$ and 3,000 to $4,320 \mathrm{~cm}^{-1}$ ) have located a number of lines, of calculated intensity down to 0.001 , that belong to the $R_{2,1}$ and $P_{\overline{2}_{1}}$ branches of the levels listed here. A few of the stronger lines of the $R_{4,3}$ branch also appear in the solar absorption. It is of further interest to note that in the solar absorption curve obtained by Migeotte on the Jungfraujoch, the relative intensity of the lines between 3,900 to 4,020 $\mathrm{cm}^{-1}$ differs from our laboratory observations, in that on his tracings the lines of the $R_{2, i}$ series are stronger relative to the $R_{0,1}$ series than on ours. This is to be expected, in view of the much lower temperature (surface temperature $\sim 257^{\circ} \mathrm{K}$ ) prevailing on the Jungfraujoch. As the $R_{0,1}$ lines in a given region of the spectrum originate on much higher energy levels than the $R_{2, \overline{1}}$ lines in the same region, there is a pronounced temperature coefficient of their relative intensity, as observed. Indeed, it would seem possible to use this effect as a thermometric device, accurate to a few degrees.

The essential correctness of the rotational analysis may also be shown by a comparison of the energy levels of table 2 with those calculated from the theory of the asymmetric rotor. This calculation is relatively simple [15], if one ignores both the influences of centrifugal distortion and of the $\nu_{1}-\nu_{3}$ perturbation. There should then be three constants, the effective reciprocal moments of inertia, $A_{v}>B_{v}>C_{v}$, in terms of which the energies may be calculated. A procedure by which these constants may be evaluated from the energy levels, together with some of the constants that characterize the centrifugal stretching, has been developed [16] and will be described in detail elsewhere. The method (which is an extension of that suggested by Mecke [1]) consists in taking certain sums and differences of the observed energy levels, grouped according to the levels of like symmetry type for each $J$, and dividing by appropriate 

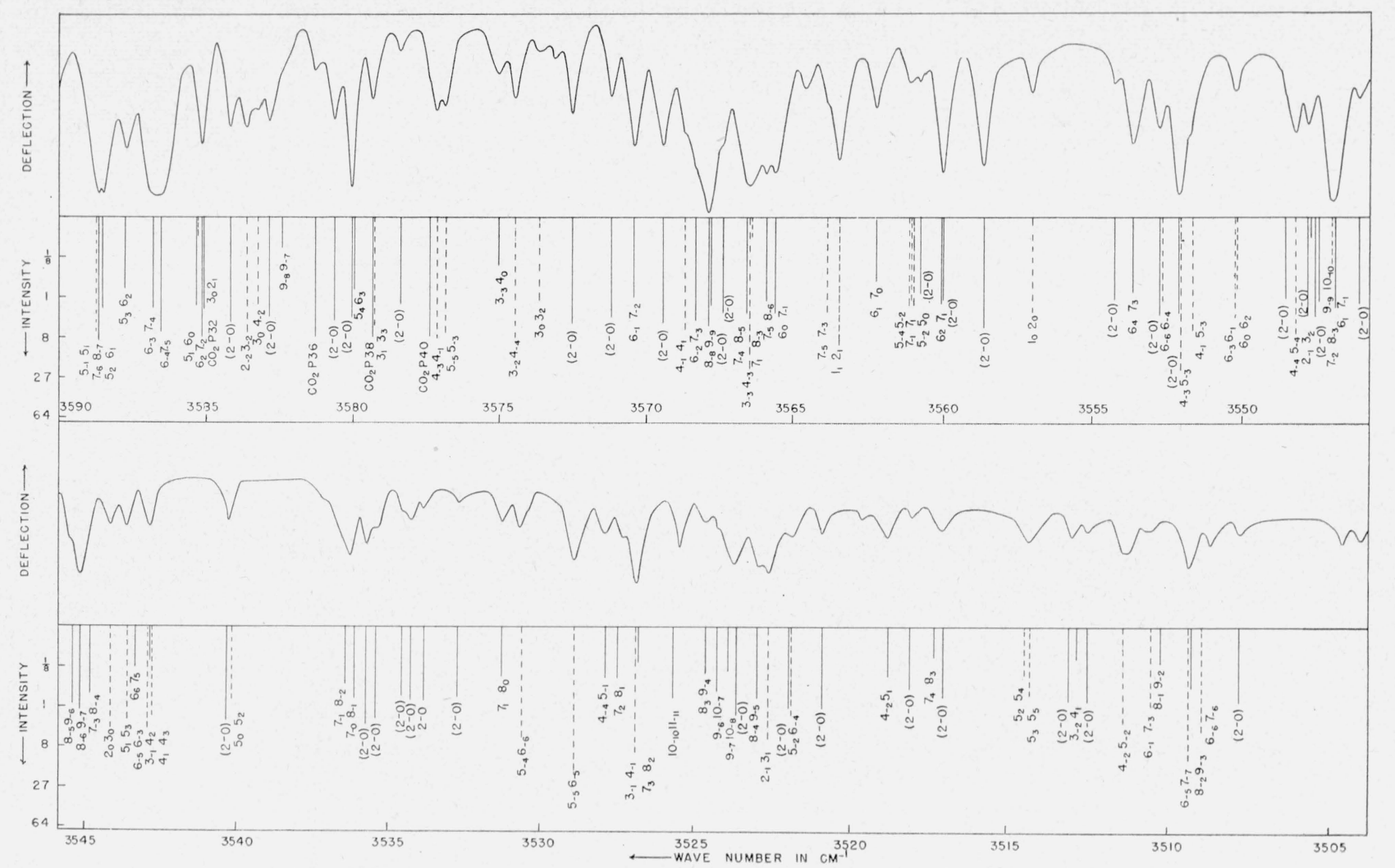

Figure 8. Comparison of observed and theoretically calculated spectra, 3,590 to $3,505 \mathrm{~cm}^{-1}$.

functions of $J$. Each such operation yields a parameter that we designate $A^{\prime}{ }_{v}(J), A^{\prime \prime}{ }_{v}(J), A^{\prime \prime \prime}{ }_{v}(J)$, etc., and which, if there were no centrifugal stretching nor perturbation, should be the desired constant $A_{v}$, etc. If there is no perturbation, each such set, when plotted against $J^{2}$, should fall on smooth curves, whose zero intercept is close to $A_{v}$, and whose initial slope yields one of the centrifugal stretching constants. The three sets, denoted by ', ', and "' do not coincide precisely, but may be brought into coincidence by making a correction involving one of the minor centrifugal stretching constants, and plotting against $J^{2 *}$, which takes the value $J^{2}-J, J^{2}-1$, or $J^{2}$, depending upon the particular set.

In figure 9 the values of the parameters, obtained in the above manner from the data of table 2, are plotted against $J^{2 *}$. The data for the ground state (000), which are obtained from many observed bands, are by far the most precise; it is seen that they fall excellently on smooth, nearly linear curves. (As the quantities plotted are of the order of $\Delta E / J^{2}$, it follows that an error of $0.5 \mathrm{~cm}^{-1}$ in any single energy at $J=7$ would lead to a deviation of 0.01 , a scatter which the (000) curves do not exceed). The effective moments of the ground state are believed accurate to $\pm 0.005 \mathrm{~cm}^{-1}$. The points for the vibrationally excited levels, denoted by the filled symbols, show much greater scatter from smooth curves in figure 9. This is to be expected, because of the less accurate data, and particularly because of the vibrational-rotational perturbation. The latter may be computed by an approximate calculation, to be described presently; from the "unperturbed" levels obtained by subtracting the corrections from the observed levels, new values of the parameters may be calculated. These are represented by the open symbols in figure 6 . They show considerably less deviation from the smooth curves that have been drawn to fit them. In drawing the curves, the slopes for $B$ and $C$ were drawn approximately parallel to those for $(000)$, as similar treatments of other vibrational levels has shown that these do not vary widely with the vibrational state. The slope of the $A$ curves are somewhat lower for the excited states than for $(000)$, again in conformity with the general finding. From the curves as drawn, values of the effective moments were obtained and are listed in table 5. These are believed accurate to about $\pm 0.02 \mathrm{~cm}^{-1}$. The value of $C_{100}+C_{001}$ does not involve the perturbation corrections, and should be somewhat more accurate.

From the values of the effective rotational constants, the value of each energy level may now be calculated and compared with the observed energy. This is done for the levels up to $J=9$ ( $\nu_{1}$ up to $J=6$ ) in table 6 . The first entry is the difference between the calculated and the observed energy; this is the 
TABLE 4. Series regularities in the $R_{0,1}$ branch of $\nu_{2}$

\begin{tabular}{|c|c|c|c|c|c|c|c|c|c|c|c|c|c|c|c|c|c|}
\hline$J^{1}$ & $J+\tau$ & 0 & 1 & 2 & 3 & 4 & 5 & 6 & 7 & 8 & 9 & -10 & 11 & 12 & $13-14$ & $15-16$ & $17-18$ \\
\hline 5 & 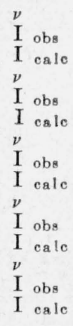 & $\begin{array}{c}3779.41 \\
99 \\
10.0 \\
3801.15 \\
100 \\
52.0 \\
3820.65 \\
100 \\
19.9 \\
3837.99 \\
100 \\
55.0 \\
(3854.3) \\
(100) \\
14.4\end{array}$ & $\begin{array}{c}3796.13 \\
99 \\
12.3 \\
3815.96 \\
100 \\
52.0 \\
3835.02 \\
100 \\
17.3 \\
3852.29 \\
100 \\
42.4\end{array}$ & $\begin{array}{c}3806.74 \\
100 \\
35.0 \\
3831.68 \\
99 \\
15.9 \\
(3854.3) \\
(100) \\
43.7 \\
3874.53 \\
100 \\
10.06\end{array}$ & \begin{tabular}{|c|}
3821.70 \\
100 \\
24.2 \\
3843.89 \\
99 \\
9.83 \\
3865.22 \\
100 \\
24.8
\end{tabular} & $\begin{array}{c}3826.66 \\
95 \\
8.04 \\
(3854.3) \\
(100) \\
28.9 \\
3880.07 \\
100 \\
7.76\end{array}$ & \begin{tabular}{|c|}
3840.11 \\
98 \\
3.79 \\
3861.87 \\
98 \\
12.2
\end{tabular} & \begin{tabular}{|c|}
3841.22 \\
98 \\
10.13 \\
3864.48 \\
90 \\
4.06
\end{tabular} & \multicolumn{2}{|c|}{$\begin{array}{c}3857.43 \\
95 \\
5.21\end{array}$} & \multirow{2}{*}{\multicolumn{2}{|c|}{$\begin{array}{r}3871.65 \\
65 \\
1.35 \\
3894.13 \\
(70) \\
1.12\end{array}$}} & \multirow{2}{*}{\multicolumn{2}{|c|}{$\begin{array}{r}3884.08 \\
30 \\
0.32\end{array}$}} & & & \\
\hline 7 & $\begin{array}{l}\nu \\
I_{\text {obs }} \\
\mathrm{I}_{\text {calo }} \\
\nu \\
\mathrm{I}_{\text {obs }} \\
\mathrm{I} \text { oale }\end{array}$ & \begin{tabular}{|c|}
3870.21 \\
100 \\
30.0 \\
3886.08 \\
100 \\
6.10
\end{tabular} & $\begin{array}{c}3869.37 \\
100 \\
9.91 \\
3885.77 \\
100 \\
18.2\end{array}$ & $\begin{array}{c}3891.30 \\
100 \\
19.4 \\
3906.15 \\
90 \\
3.47\end{array}$ & $\begin{array}{c}3880.33 \\
100 \\
5.60 \\
3899.38 \\
(100) \\
9.62\end{array}$ & \begin{tabular}{|c|}
3904.22 \\
100 \\
14.6 \\
3925.20 \\
88 \\
2.49
\end{tabular} & $\begin{array}{c}3883.27 \\
80 \\
2.98 \\
3904.22 \\
(100) \\
5.18\end{array}$ & $\begin{array}{c}3899.38 \\
(100) \\
8.75 \\
3925.20 \\
(88) \\
1.63\end{array}$ & $\begin{array}{c}3880.07 \\
(100) \\
1.25 \\
3902.15 \\
98 \\
2.41\end{array}$ & \begin{tabular}{|c|}
3881.05 \\
95 \\
3.74 \\
3905.51 \\
60 \\
0.80
\end{tabular} & & & & & & & \\
\hline 10 & $\begin{array}{l}\nu \\
I_{\text {obs }} \\
I_{\text {ealo }} \\
\nu \text { a } \\
I_{\text {obs }} \\
I_{\text {calc }} \\
\nu{ }^{2} \\
I_{\text {obs }} \\
I_{\text {cale }}\end{array}$ & $\begin{array}{r}3901 . \\
100 \\
13 . \\
3917 . \\
92 \\
6 . \\
3932 . \\
85 \\
2 .\end{array}$ & & \begin{tabular}{|c}
3920.12 \\
90 \\
4. 99 \\
3934.25 \\
55 \\
0.71 \\
3948.37 \\
55 \\
0.84
\end{tabular} & $\begin{array}{c}3916.35 \\
80 \\
1.61 \\
3932.42 \\
88 \\
2.12 \\
3947.39 \\
55 \\
0.28\end{array}$ & \begin{tabular}{|c}
3942.86 \\
90 \\
3.25 \\
3957.05 \\
35 \\
0.41 \\
3969.38 \\
42 \\
0.42
\end{tabular} & \begin{tabular}{|c|}
3924.38 \\
60 \\
0.84 \\
3942.86 \\
$(90)$ \\
1.05 \\
3959.89 \\
18 \\
0.128
\end{tabular} & \begin{tabular}{|c|}
3950.12 \\
80 \\
2.23 \\
3972.16 \\
28 \\
0.28 \\
3990.72 \\
28 \\
0.27
\end{tabular} & \begin{tabular}{|c|}
3923.70 \\
40 \\
0.41 \\
3944.49 \\
45 \\
0.51 \\
3963.99 \\
10 \\
0.055
\end{tabular} & \begin{tabular}{|c|}
3930.83 \\
65 \\
1.20 \\
3958.43 \\
20 \\
0.165 \\
3994.78 \\
10 \\
0.166
\end{tabular} & $\begin{array}{c}3916.35 \\
(80) \\
0.16 \\
3938.42 \\
25 \\
0.217 \\
3959.89 ? \\
(18) \\
0.027\end{array}$ & $\begin{array}{c}3917.32 \\
(92) \\
0.48 \\
3940.80 \\
6 \\
0.072 \\
3964.90 \\
12 \\
0.079\end{array}$ & \multicolumn{2}{|c|}{$\begin{array}{c}3906.15 \\
(90) \\
0.20 \\
3928.19 \\
15 \\
0.101 \\
3950.51 ? \\
(25) \\
0.040\end{array}$} & \begin{tabular}{|c|}
3895.18 \\
8 \\
0.041 \\
$(3916.35) ?$ \\
$(80)$ \\
0.028
\end{tabular} & 0.005 & 0.005 \\
\hline 11 & $\begin{array}{l}\nu \\
I_{\text {obs }} \\
\text { I calc }\end{array}$ & $\begin{array}{r}3947 . \\
60 \\
1 .\end{array}$ & & $\begin{array}{c}3962.11 \\
(48) \\
0.098\end{array}$ & $\begin{array}{c}3961.92 \\
50 \\
0.291\end{array}$ & $\begin{array}{c}3979.68 ? \\
8 \\
0.040\end{array}$ & $\begin{array}{c}3975.10 \\
20 \\
0.123\end{array}$ & $\begin{array}{c}4005.52 ? \\
3 \\
0.026\end{array}$ & $\begin{array}{c}3982.05 ? \\
15 \\
0.057\end{array}$ & $\begin{array}{c}4019.52 ? \\
(15) \\
0.016\end{array}$ & $\begin{array}{c}3980.75 ? \\
6 \\
0.025\end{array}$ & .008 & \begin{tabular}{|r|}
$3975.77 ?$ \\
3 \\
0.010
\end{tabular} & .003 & .004 & .001 & .0003 \\
\hline 13 & $\begin{array}{l}\nu \\
I_{\text {obs }} \\
\mathrm{I}_{\text {calo }} \\
\nu_{\mathrm{I}} \\
\mathrm{I}_{\text {obs }} \\
\mathrm{I} \text { calo }\end{array}$ & $\begin{array}{r}3962 . \\
48 \\
0 . \\
3976 . \\
26 \\
0 .\end{array}$ & $\begin{array}{l}11 \\
368 \\
26 \\
116\end{array}$ & $\begin{array}{r}3976 . \\
10 \\
0 . \\
3989 . \\
10 \\
0 .\end{array}$ & $\begin{array}{l}49 \\
125 \\
56 ? \\
034\end{array}$ & .036 & .012 & $\begin{array}{c}4018.59 ? \\
(5) \\
0.019 \\
.002\end{array}$ & .005 & $\begin{array}{c}4031.55 ? \\
5 \\
0.012\end{array}$ & .002 & .006 & .001 & .003 & .0012 & .0004 & \\
\hline 15 & $\begin{array}{l}\nu \\
I_{\text {obs }} \\
I_{\text {calo }} \\
\nu \\
I_{\text {obs }} \\
I_{\text {calo }}\end{array}$ & $\begin{array}{r}3990 . \\
15 \\
0 . \\
4007 . \\
6 \\
0 .\end{array}$ & $\begin{array}{l}22 ? \\
.032 \\
57 ? \\
008\end{array}$ & $\begin{array}{r}4005 . \\
3 \\
0 .\end{array}$ & $\begin{array}{l}53 ? \\
009\end{array}$ & .00 & & .001 & & & & & & & & & \\
\hline
\end{tabular}

combined result of the effects of centrifugal distortion and perturbation, together with any errors resulting from experimental inaccuracy in locating the levels, or in deriving the rotational constants. It is seen that the differences are roughly alike for the three vibrational states, and increase in a regular manner with $J$ and with $J+\tau$, except for the more strongly perturbed levels, which have been given letter suffixes.

The second number entered in table 6 for each rotational level of $\nu_{1}$ and $\nu_{3}$ is an estimate of the energy shift due to the perturbation. These are, of course, of equal magnitude and opposite sign in $\nu_{1}$ and $\nu_{3}$ for each symmetry group of each $J$. They were obtained by the following approximate procedure. An exact calculation [13] would involve setting up and solving secular determinants of order $J$ or $J+1$, in which the elements are the usual rotational energy constants of the Wang determinant, plus perturbation elements of the form $W_{p}=G_{x x}$ $(J+K+1)(J-K) \cdot G_{x x}$ is a constant that depends on the potential constants of the molecular motion and on the vibrational wave functions of $\nu_{1}$ and $\nu_{3}$. It might be calculated from the potential function,
TABLE 5. Rotational constants for $\mathrm{H}_{2} \mathrm{O}$ bands

\begin{tabular}{|c|c|c|c|c|c|c|c|c|c|}
\hline$v_{1}$ & $v_{2} \quad v_{3}$ & $A_{\sigma}$ & $B$, & $C$, & $\kappa$ & $10^{40} I_{A}$, & $10^{40} I_{B}$ & $10^{40} I_{C_{\mathrm{v}}}$ & $\Delta$ \\
\hline & & $\mathrm{cm}^{-1}$ & $\mathrm{~cm}^{-1}$ & $m^{-1}$ & & $g \mathrm{~cm}^{2}$ & $g \mathrm{~cm}^{2}$ & $g \mathrm{~cm}^{2}$ & \\
\hline 0 & & 27.877 & 14. 512 & 9. 285 & -0.4377 & 1.004 & 1. 928 & 3. 014 & 0.082 \\
\hline 1 & $\begin{array}{ll}0 & 0\end{array}$ & 27.13 & 14.29 & 9. 105 & -.4247 & 1. 031 & 1. 958 & 3. 073 & .084 \\
\hline 0 & $\begin{array}{ll}0 & 1\end{array}$ & 26.636 & 14.40 & 9.156 & -.4000 & 1. 051 & 1. 913 & 3. 056 & .062 \\
\hline
\end{tabular}

but is better treated as an empirical constant, inasmuch as it is found [4] to vary with the particular pair of interacting levels; that is, it has a different value in the interacting combination bands (011-110); (021-120); (111-210), etc., than is found here. It is now assumed that each rotational level is perturbed by each interacting level $K \pm 1$ separately, so that the perturbed energy may be given by $E=E_{0}+\sum_{i} \delta_{i}$ where $E_{0}$ is the "unperturbed" energy (such as would result from a solution of each Wang secular equation, with appropriate centrifugal stretching terms), and

$$
\delta_{i}=\frac{\left\{\left(E_{0}-E_{i}\right)^{2}+W_{p i}^{2}\right\}^{1 / 2}-\left(E_{0}-E_{i}\right)}{2}
$$




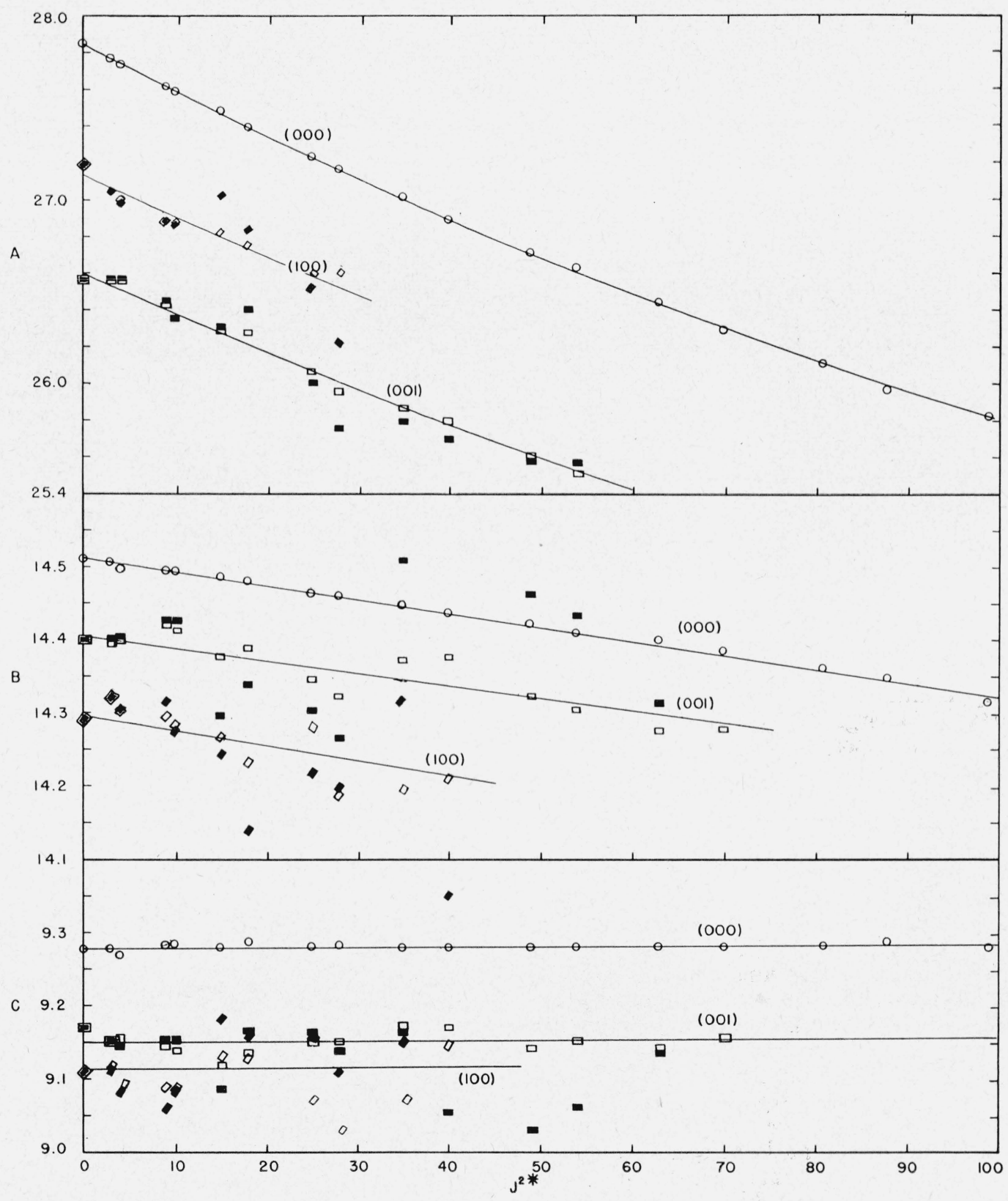

FIGURE 9. Curves from which may be derived the inertial and centrifugal-stretching constants, associated with rotation about the least axis of inertia $(A)$, the intermediate axis $(B)$, and the greatest axis $(C)$.

$\bigcirc$, Ground vibrational state (000); $\downarrow \nu_{1}(100)$, uncorrected for perturbation; $\diamond, \nu_{1}(109)$, corrected for perturbation; $\mathbf{R}, \nu_{3}(001)$, uncorrected for perturbation; $\square,{ }_{3} \nu(001)$, corrected for perturbation. 
TABLE 6. Centrifugal and perturbation energies $\left(\mathrm{cm}^{-1}\right)$

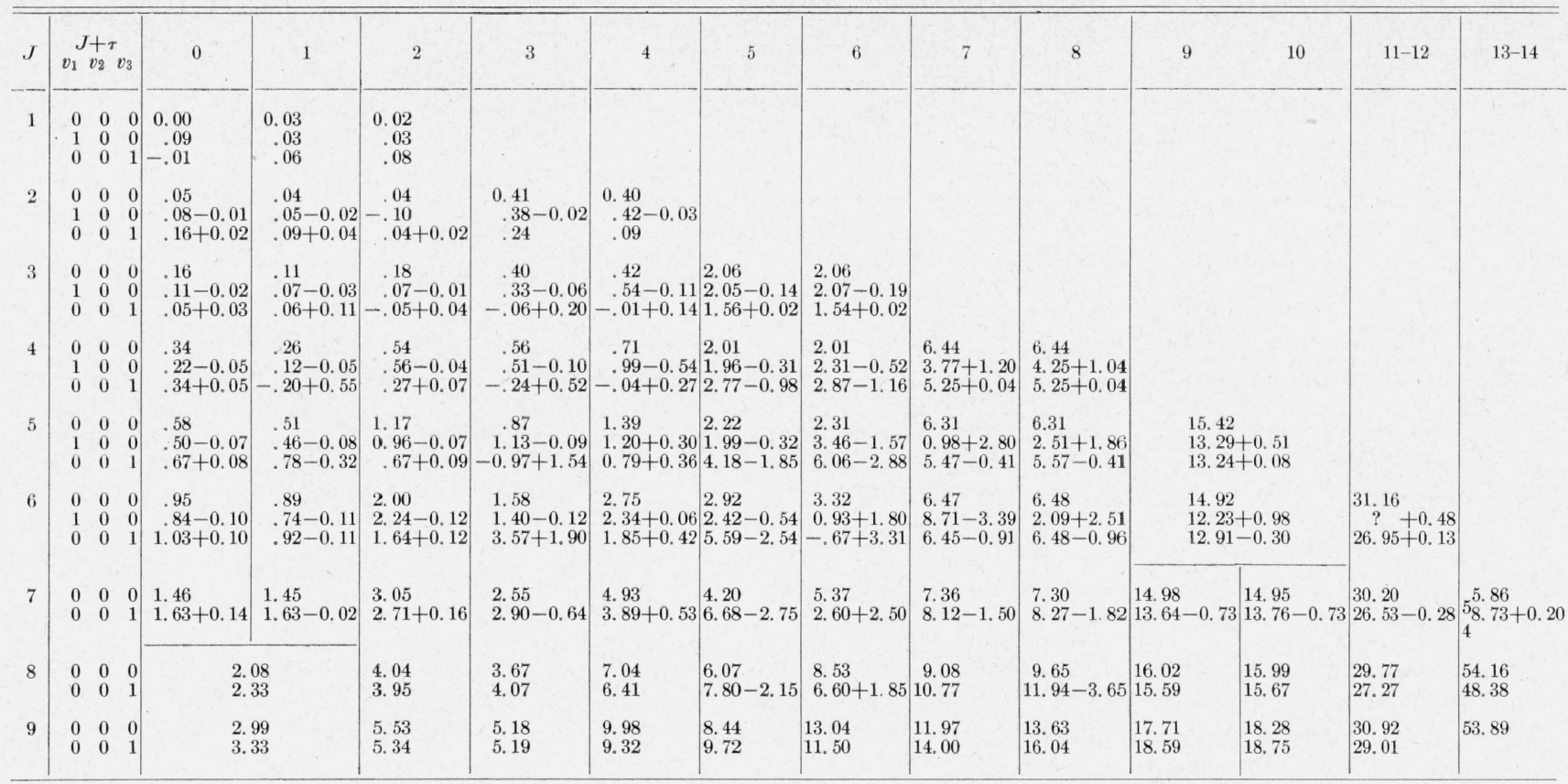

$E_{i}$ being the unperturbed energy of the neighboring level, with the perturbation parameter $W_{p i}$. This is the usual first-order perturbation formulation; and tests with a few secular equations of low order have shown it to be a good approximation to the more complicated exact procedure, when as is the case for these states, there is only one level $E_{i}$ close to $\mathrm{E}_{0}$.

With the approximate procedure, and an estimate for the unperturbed energies obtainable by inspection from table 6 , it is thus possible to calculate from the observed levels a value of $W_{p}$ and hence $G_{x x}$ for each strong perturbation. The values thus obtained for $G_{x x}$ do not agree well, showing a quite regular tendency to increase with higher $K$. This might mean that the Coriolis interaction is augmented by the centrifugal distortion associated with rotation about the least axis of inertia, which is not surprising. By assuming for $G_{x x}$ the value $1.00+0.65 K(K+1)$, we obtain the perturbation corrections listed in table 6 . It is seen that these produce a gratifying smoothing of the entries, so that the sums, which now should be due to centrifugal stretching alone, vary quite regularly with $J$ and $J+\tau$. Without further calculation, one should not ascribe great significance to this empirical perturbation correction, but it appears to yield results, for low $J$ at least, that are quite reasonable, and further confirms the energy levels and molecular constants reported here.

The very small residuals in table 6 for the lowest $J$ values may also be taken as confirmation of the values of the band origins listed in table 2. Except for some systematic error that would affect all our frequencies, they are probably accurate within $\pm 0.05 \mathrm{~cm}^{-1}$. The $0_{0}$ level of $\nu_{1}$ is not directly observed, as the only line to which it would lead $\left(0-1_{0}\right.$, at $3619.92 \mathrm{~cm}^{-1}$ ) is overlapped by strong lines of $\nu_{3}$ and of $\mathrm{CO}_{2}$, but the consistency of the other $\nu_{1}$ lines and levels here reported establishes it with good accuracy.

The lower levels of $\nu_{3}$ here identified agree with those reported earlier [3]. However, beginning at $J=4$ there are numerous differences. Our $\nu_{1}$ levels do not agree in any particular with the earlier analysis. It may be noted that the present results for $\nu_{1}$ are in agreement with the frequency shifts observed in the Raman effect [17], when the vibrational-rotational selection rules applicable to the latter are considered. The Raman observations yield a broad unresolved "line" with two maxima at $3646.1 \pm 1.5$ and $3653.9 \pm$ $1.5 \mathrm{~cm}^{-1}$. The selection rules [18] show that this is really an unresolved $Q$ branch in which are superposed all lines with $\Delta J=0, \Delta \tau=0$. For each $J$, the strongest lines are those with the highest and lowest $\tau$. Because of the lower rotational constants in the upper vibrational state, the shifted lines will be below the band origin of $3657.05 \mathrm{~cm}^{-1}$. The center of gravity of the unresolved band may be approximately calculated from the energy levels of table 2, with the unobserved higher levels estimated from the rotational constants. Such a calculation, assuming a scattering temperature of $150^{\circ} \mathrm{C}$ and an effective slit width of $3 \mathrm{~cm}^{-1}$ leads to the observed doublet structure, in which the higher frequency shifts are due principally to the lines of lowest $\tau$, with maxima at $3654.3 \mathrm{~cm}^{-1}$ and $3647.5 \mathrm{~cm}^{-1}$.

\section{Conclusion}

With the present identification of $\nu_{1}$, and the derivation of quite accurate rotational constants for $\nu_{1}$ and $\nu_{3}$, the last significant gap in our knowledge of 
the vibration-rotation spectrum of $\mathrm{H}_{2} \mathrm{O}$ is filled. Additional studies at elevated temperature would undoubtedly be of value in establishing higher rotational term values, and improved resolution, by splitting some of the still blended lines, might lead to a slight revision in the energy levels. Moreover, studies in the region 3,300 to 3,600 will undoubtedly permit extension of the $\nu_{1}$ analysis. With an asymmetric rotor of the complexity of $\mathrm{H}_{2} \mathrm{O}$, one can never consider the spectral analysis complete. Nevertheless, it is believed that the atmospheric absorption in the $2.7-\mu$ region is now rather satisfactorily accounted for.

Our thanks are due to N. Acquista and Mary A. Lamb, who assisted in obtaining and measuring the spectra. We are also indebted to O. C. Mohler of the Mc-Math Hulbert Observatory, University of Michigan, to W. A. Benesch of the Johns Hopkins University, and to M. Migeotte of Liége, Belgium, for communicating their results on the atmospheric absorption, which were of assistance in interpreting the data.

\section{References}

[1] R. Mecke, Z. physik 81, 373 (1933)

[2] E. K. Plyler and W. W. Sleator, Phys. Rev. 37, 1493 (1931).
[3] H. H. Nielsen, Phys. Rev. 62, 422 (1942).

[4] W. S. Benedict, in preparation; of Phys. Rev. 74, 702 , 703, 1246 (1948).

[5] E. K. Plyler and M. A. Lamb, National Bureau of Standards, publication pending.

[6] E. F. Barker, Astrophys. J. 55, 391 (1922).

[7] R. C. Nelson, The absorption of water vapor in the regions 1.33 to 1.48 and 1.77 to 1.98 microns, Summary Report No. 4 on Contract Nobs 28373 (Northwestern Univ., May 23, 1949).

[8] P. C. Cross, R. M. Hainer, and G. W. King, J. Chem. Phys. 12, 210 (1949). 1944

[9] D. M. Dennison, Rev. Mod. Phys. 12, 189 (1940).

[10] G. Herzberg, Infra red and raman spectra of polyatomic molecules, p. 390 (D. Van Nostrand, New York, N. Y., 1945).

[11] L. Goldberg, O. C. Mohler, R. R. MeMath, and A. K Pierce, Phys. Rev. 76, 1948 (1949); also G. Herzberg, private communication.

[12] Plyler and Sleator, Phys. Rev. 37, 1493 (1931); H. H. Nielsen, Phys. Rev. 59, 565 (1941); W. A. Benesch, private communication; M. Migeotte, private communication.

[13] E. B. Wilson, J. Chem. Phys. 4, 313 (1936); also [10], p. 466.

[14] R. M. Hainer and G. W. King, J. Chem. Phys. 15, 89 (1947).

[15] G. W. King, R. M. Hainer, and P. C. Cross, J. Chem. Phys. 11, 27 (1943)

[16] W. S. Benedict, Phys. Rev. 75, 1317 (1949).

[17] D. H. Rank, K. D. Larsen and E. B. Bordner, J. Chem. Phys. 2, 464 (1934).

[18] G. Placzek and E. Teller, Z. Physik, 81, 209 (1933).

Washington, July 19, 1950. 